\title{
Spatial clustering of inhibition in mouse primary visual cortex
}

\author{
Pawan Bista ${ }^{1,2}$, Rinaldo D. D'Souza ${ }^{1,2}$, Andrew M. Meier ${ }^{1}$, Weiqing Ji ${ }^{1}$, Andreas Burkhalter ${ }^{1}$ \\ ${ }^{1}$ Department of Neuroscience, Washington University School of Medicine, St. Louis, Missouri, \\ 63110, ${ }^{2}$ Co-first author
}

Keywords: visual cortex; inhibition; parvalbumin interneurons; thalamocortical connections; intracortical feedback

Correspondence:

Andreas Burkhalter PhD

Department of Neuroscience, 8108

Washington University School of Medicine

660 S. Euclid Avenue

St. Louis, MO 63110

Phone: (314) 362-4068

Fax: (314) 362-3446

E-mail: burkhala@wustl.edu 


\section{SUMMARY}

Whether mouse visual cortex contains orderly feature maps is debated. The overlapping pattern of geniculocortical (dLGN) inputs with M2 muscarinic acetylcholine receptor-rich patches in layer 1 (L1) suggests a non-random architecture. Here, we found that L1 inputs from the lateral posterior thalamus (LP) avoid patches and target interpatches. Channelrhodopsin-assisted mapping of EPSCs in L2/3 shows that the relative excitation of parvalbumin-expressing interneurons (PVs) and pyramidal neurons (PNs) by dLGN, LP and cortical feedback are distinct and depend on whether the neurons reside in clusters aligned with patches or interpatches. Paired recordings from PVs and PNs shows that unitary IPSCs are larger in interpatches than patches. The spatial clustering of inhibition is matched by dense clustering of PV-terminals in interpatches. The results show that the excitation/inhibition balance across V1 is organized into patch and interpatch subnetworks which receive distinct long-range inputs and are specialized for the processing of distinct spatiotemporal features.

\section{INTRODUCTION}

The modernist's maxim that form follows function manifests itself in neuroscience as functional architecture. The mesoscale description of the spatial relationship between neuronal responses, layers, columns and areas, has driven much of what is known about the matrix of the cortical machinery (Hubel and Wiesel, 1962, 1974). The concepts on the network organization that emerged from these foundational studies include: the orderly representation of space in topographic maps, the complexification of receptive fields across layers, functionally distinct columns, distributed hierarchical processing along specialized functional streams, the exponential distance rule of cortical connectivity, and the selection of sensory input based on feedback from higher areas (Felleman and Van Essen, 1991; Horvát et al., 2016; Hubel and Wiesel, 1962). Although the functional architecture implies that the spatiotemporal tuning of cell populations is determined by their connectivity and physical layout, the underlying networks of modular processing have been difficult to define (DaCosta and Martin, 2013). The challenge has been greatest in rodents because of the long-held view that cortex lacks columnar organization (Ohki et al., 2005; 2007). However, recent studies in mouse primary visual cortex (V1) have found that subcortically and intracortically projecting PNs are spatially clustered and are vertically aligned to mini- and microcolumns of cell bodies and dendrites with distinct tuning preferences (Kondo et al, 2016; Marukoda et al., 2017; Znamenskiy et al. 2018). 
Previously, we found a fixed interdigitating pattern of M2 muscarinic acetylcholine receptorexpressing $(\mathrm{M} 2+)$ patches and $\mathrm{M} 2$ - interpatches in L1 of mouse V1, which aligns with functionally distinct cell clusters in L2/3 tuned for high spatial frequency (SF) and high temporal frequency (TF), respectively (Ji et al., 2015). This finding provides structural evidence for functionally discrete modules and raises the question whether the excitation (E) / inhibition (I) balance in patches and interpatches with diverse spatiotemporal stimulus preferences is spatially organized. That inhibition is not a uniform blanket across V1 (Karnani et al., 2014), but is deployed in spatially clustered patterns by subtypes of PV or somatostatin- (SOM) expressing GABAergic neurons (Ebina et al., 2014; Maruoka et al., 2017), and that activation of these cells including those which express vasoactive intestinal peptide can shape stimulus selectivities of PNs is gradually gaining acceptance (Ayzenshtat et al., 2016; Lee, et al., 2012; Lee et al. 2014; Zhu et al., 2015). Whether the inhibitory network is tied to the spatially clustered patch/interpatch system in V1 and provides distinct subnetworks for processing visual information remains unknown.

To determine whether inhibition across $\mathrm{V} 1$ is modular we measured the strength of synaptic long-range input to L2/3 PNs and PVs in patches and interpatches by using subcellular Channelrhodopsin-2 (ChR2) assisted circuit mapping (sCRACM) (Mao et al, 2011; Petreanu et al., 2009). We recorded from pairs of PVs and PNs and analyzed unitary excitatory (uEPSCs) and inhibitory (uIPSCs) postsynaptic currents in patches and interpatches. The results show that $\mathrm{V} 1$ contains two different circuit motifs in which patches and interpatches have distinct thalamocortical and interareal inputs to PNs and PVs, and that PVs in interpatches provide stronger feedforward inhibition (FFI) to PNs than in patches. The modular organization of inhibition is consistent with the notion that neurons in interpatches are more sensitive to rapidly changing visual inputs (Ji et al., 2015).

\section{RESULTS}

\section{Clustering of thalamocortical inputs to L1 of V1}

We have recently shown that inputs from the dLGN to L1 of V1 are spatially clustered and overlap with the patchy pattern of M2 immunostaining (Ji et al., 2015). Here, we show in tangential sections of flatmounted cortex of Chrm2tdT mice that $\mathrm{dLGN} \rightarrow \mathrm{V} 1$ axons labeled by anterograde tracing with AAV2/1.hSyn.EGFP preferentially terminate in $M 2+$ patches of $L 1$ and avoid M2interpatches (Figure 1A-C). For quantitative analysis we divided images into patches and interpatches based on M2 fluorescence intensity contour maps (Sincich and Horton, 2005) (Figure 
S1), and measured EGFP intensity in patches (top $3^{\text {rd }}$ ) and interpatches (bottom $3^{\text {rd }}$ ). We then normalized the pixel values in interpatches to the mean intensity in patches and plotted the counts in different intensity bins. We found that the fluorescence intensity in patches was $2.1 \pm 0.024$ fold ( $p=8 \times 10^{-18}$, Komolgorov-Smirnov test [KS]) higher in patches than interpatches (Figure 1D). Similar to our previous findings, patches and interpatches in $\mathrm{L} 1$ were 60-80 $\mu \mathrm{m}$ wide and their centroids were 120-140 $\mu \mathrm{m}$ apart. dLGN projections to L4, and 5/6 appeared uniform (data not shown). Inputs to L1 from the LP thalamus exhibited a strikingly different pattern, showing $1.6 \pm$ 0.14-fold $\left(p=1.33 \times 10^{-4}, \mathrm{KS}\right)$ stronger projections to $\mathrm{M} 2$ - interpatches (Figure 1E-H). Simultaneous tracing of dLGN and LP inputs with AAV2/1hSyn.tdTomato.WPRE.bGH and AAV2/1.hSyn.EGFP, respectively, confirmed the interdigitating pattern of projections from primary and higher order thalamic nuclei, showing denser LP input to interpatches $\left(p=7.95 \times 10^{-4}, \mathrm{KS}\right)$ (Figure 1I-M).

\section{Clustering of intracortical inputs to L1 of V1}

We next compared feedback projections from the higher visual cortical ventral stream lateromedial area, LM, to V1 with inputs from the dLGN. Double viral tracings from the dLGN (AAV2/1.hSyn.EGFP) and LM (AAV2/1hSyn.tdTomato.WPRE.bGH) showed that inputs from both sources overlapped in presumtive $\mathrm{M} 2+$ patches of $\mathrm{L} 1$ (Figure $1 \mathrm{~A}-\mathrm{C} ; 2 \mathrm{~A}$ ). Quantitative analysis showed that LM inputs to patches were $1.7 \pm 0.05$-fold denser than to interpatches $\left(p=1.45 \times 10^{4}\right.$, KS) (Figure 2B). We have shown previously that inputs from the dorsal stream anterolateral area, $\mathrm{AL}$, terminate in $\mathrm{M} 2+$ patches (Ji et al., 2015), raising the question whether $\mathrm{M} 2+$ patches are the preferred targets of cortical feedback. To address this, we traced the connections to V1 from the posteromedial area, PM, another member of the dorsal stream (Wang et al. 2012). We found that inputs from PM were non-uniform, overlapped in part with patches and interpatches but avoided the top and bottom intensity quantile of each compartment (Figure 2F-G). The spatial distribution of $\mathrm{PM} \rightarrow \mathrm{V} 1$ inputs showed no difference between patches and interpatches (Figure $2 \mathrm{H}$ ). However, simultaneous labeling of inputs from $\mathrm{AL}$ and PM showed an interdigitating pattern and revealed a fine-scale pattern in patches (Figure $2 \mathrm{I}-\mathrm{L}$ ). This demonstrates that projections from $\mathrm{AL}$ targets the top and PM the bottom intensity quantile of patches and indicates that the terminal patterns of feedback to $\mathrm{V} 1$ by the two dorsal stream areas is not identical.

\section{Clustering of cholinergic inputs to L1 of V1}

Classic studies suggest that the cholinergic innervation of cortex is diffuse, signaling is through volume transmission, and that spatial selectivity arises from spatial clustering of receptors and 
axons (Muñoz and Rudy, 2014). But whether cholinergic fibers in cortex are spatially mapped in systematic fashion is unknown. To address the question we stained tangential sections through V1 with antibodies against M2 and choline acetyl transferase ChAT. We found that ChAT labeled axons in L1 were clustered and significantly $(p=0.009)$ denser in M2+ patches (Figure S2A-D).

\section{Development of M2 clusters}

Motivated by the report of Maruoka et al., (2017) that L5 neurons in V1 of 6-day-old (P6) mice form $20 \mu \mathrm{m}$-wide microcolumns, we have looked for clustered M2 expression in early postnatal development. Our results in tangential sections through V1 of P4 Chrm2tdT mice show that M2 expression in L1 is patchy (10 - $30 \mu \mathrm{m}$ wide, 52-79 $\mu \mathrm{m}$ center-to-center, Figure S3A). In L2/3 patches were smaller and contained multiple 0.8-1.5 $\mu \mathrm{m}$ wide rings, presumably representing membranes of cross-sectioned ascending dendrites (Figure S3B) (Kondo et al., 2016). At P10 the $L 1$ patches were larger (30 - $60 \mu \mathrm{m}$ wide) and more widely spaced (80-100 $\mu \mathrm{m}$ center-tocenter) (Figure S3C), a difference which may be accounted for by the 1.7 fold increase in brain size (Agrawal et al., 1968).

\section{Module- and pathway-specific synaptic strengths of inputs to PNs and PVs}

We have shown previously that L2/3 neurons in interpatches of V1 are more often tuned to the direction of visual motion and respond to higher speeds and TFs than neurons in patches (Ji et al., 2015). This non-uniform distribution of selectivities suggested that the two cell clusters differentially process spatiotemporal information. FFI mediated by rapid synaptic activation of PVs (D'Souza et al., 2016) is a circuit motif shown to shape the temporal sensitivity of PNs in auditory cortex independent of stimulus adaptation (Li et al., 2014; Natan et al., 2017). Because the strength of FFI depends on the excitatory input to PNs and PVs and the latter's inhibition of PNs (Atallah et al., 2012), we compared the strength of excitatory inputs to patches and interpatches from different thalamocortical and intracortical pathways to L2/3 neurons in V1. To do this we used SCRACM in acute slices of PVtdT mice in which inputs to V1 were labeled by anterograde tracing with AAV2/1.hSyn.ChR2(H134R).eYFP.WPRE.hGH.

dLGN input in tangential slices. To select for inputs to distal dendrites of L2/3 neurons in L1-2 and optimally preserve their 3D organization, we obtained tangential slices of $\mathrm{V} 1$. Whole cell patch clamp recordings were performed from pairs of PNs and PVs ( $<50 \mu \mathrm{m}$ apart) aligned with densely ChR2-eYFP-expressing patches or sparsely labeled interpatches, visualized by anterograde viral tracing from the dLGN (Figure 1A). The locations of the recorded neurons were identified by 
intracellular filling with Alexa-594 hydrazide and post hoc immunostaining of re-sectioned slices with an antibody against M2 (Figures 3A, S4A-C). Input strength was measured by EPSCs elicited by laser stimulation of ChR2-expressing axon terminals. Blue light flashes were delivered in an $8 \times 8$ grid with $75 \mu \mathrm{m}$ spacing centered on the cell body. sCRACM mapping in the same slice of dLGN inputs to neighboring PVs and PNs showed stronger synaptic activation in patches than interpatches (Figures 3A, B, D, G), which is consistent with the dense dLGN projections to patches (Figure 1B). Direct comparison of cell pairs in patches and interpatches showed that EPSCs from PVs were larger than from PNs (Figures 3B, D). The population difference is shown in plots in which responses of cell pairs are represented by single points (Figure 3C, E). The geometric mean of $\mathrm{EPSC}_{\mathrm{PN}} / \mathrm{EPSC}_{\mathrm{PV}}$ ratios in patches and interpatches is indicated by a red line intersecting the origin of the graph. Input resistance-corrected data is shown by a blue line. The average EPSC recorded from PVs $(323.6 \pm 130 \mathrm{pA})$ in patches was 2.3-fold larger $(p<0.001, \mathrm{n}=17$ pairs, t-test) than from PNs $(140.6 \pm 81.6 \mathrm{pA})$. A similar 2.3-fold difference $(p<0.001, \mathrm{n}=17$ pairs, t-test) was found in interpatches between the more weakly responsive PVs $(147.7 \pm 91.2 \mathrm{pA})$ and PNs $(62.9 \pm$ 31.1 pA). Heatmaps of EPSCs in patches and interpatches show that responses were maximal near the cell body and decreased toward the distal tip of dendrites (Figures 3B, D). The responsive area was significantly smaller for PNs than PVs (paired t-test, $p<0.001$ ), but similar in patches and interpatches (Figure 3F). dLGN inputs to PVs evoked higher current densities $(p<0.01$, paired t-test) and significantly ( $p<0.05$, paired t-test) faster rise times of EPSCs than inputs to PNs (Figures 3F, S5A). These results show that in tangential slices the strength of synaptic activation by dLGN inputs depends on cell type, the modularity of innervation and the strong preference for module-specific branching of apical dendrites in L1-2 (Figure 1A-D, 6 B-N, S4D, E, I, J). Indeed the combined length of PN $(n=12)$ and PV $(n=12)$ dendrites in patches was 3.4fold higher $(p<0.001$, t-test $)$ in patches $(812 \pm 44 \mu \mathrm{m})$ than in interpatches $(239 \pm 29 \mu \mathrm{m})$. For cells in interpatches $(n=1)$ the dendritic length in interpatches ( $898 \pm 41 \mu \mathrm{m})$ was 9.8 -fold higher $(p<0.0001$, t-test) than in patches $(91 \pm 32 \mu \mathrm{m})$.

dLGN input in coronal slices. Although tangential slices are optimal for discriminating inputs to patches and interpatches, truncation of layers may favor stimulation of distal over basal dendrites. To control for potential preferences we obtained coronal slices of V1. To distinguish patches from interpatches we traced dLGN input to L1 with AAV2/9.CAG.ChR2.Venus. Photostimulation of ChR2-expressing dLGN inputs was performed in an $8 \times 10$ (mediolateral $x$ dorsovental) $75 \mu \mathrm{m}$ grid. Recordings were obtained from pairs of L2/3 PVs and PNs which were aligned with patches and interpatches. Although patchy dLGN Venus-labeled inputs were readily distinguishable at the 
slice surface, recordings from PNs and PVs from more than a single patch or interpatch in the same slice was challenging. Despite this caveat, which precluded recordings of PV/PN pairs from both patches and interpatches in the same slice, we found that in both module types EPSCs from PVs were lager (Figure 4A-C). Because ChR2 expression varied across animals and slices it was difficult to compare responses between experiments. But the range of response amplitudes suggests that the overall sizes of EPSCs in patches and interpatches is similar. This is consistent with the uniform distribution of dLGN inputs to L3/4 and suggests that the module-specific strength of activation we observed in tangential slices (Figure $3 C, E, G$ ) is due to the non-uniform distribution of $L 1$ inputs.

LP input in coronal slices. Coronal slices were used for recording responses from L2/3 evoked by light stimulation of virally traced ChR2-expressing inputs from LP (Figure 1F). Patches were identified by viral tracing of dLGN input to L1 (Figure 1I). In recordings from the same slice we found that EPSCs from PNs were larger in interpatches than in patches (Figure 4D). This differs from the lack of modularity observed after stimulation of dLGN inputs in coronal slices (Figure 4AC) and is consistent with the preferred innervation by LP of interpatches in L1 (Figure 1E-M). Light flashes in the LP-recipient L5A (Zhou et al., 2018) were ineffective in driving EPSCs from L2/3 PNs (data not shown). Thus, similar to patch-preferring dLGN inputs to L1 observed in tangential slices (Figure 3G), interpatch-preferring responses evoked by LP inputs in coronal slices are mediated through synapses in L1. Next, we compared the relative strengths of L1 LP-synapses in activating PNs and PVs in patches and interpatches in different slices. Unlike the strong bias for PVs observed by inputs from the dLGN (Figure 1G), we found that responses in patches and interpatches to LP inputs were matched, with a median EPSC $_{\mathrm{PN}} /$ EPSC $_{\mathrm{PV}}$ ratio in both compartments not significantly different than unity (Figure 4E-H). These results suggest that FFI in $L 2 / 3$ produced through synapses in $L 1$ is weaker in the $L P \rightarrow V 1$ than the $d L G N \rightarrow V 1$ pathway.

Feedback input from $L M$. Similar to thalamocortical pathways, input from $L M \rightarrow V 1$ terminated in periodic clusters of L1 where they largely overlapped with projections from the dLGN (Figures $2 A)$. Photostimulation of ChR2-expressing $L M \rightarrow V 1$ feedback axons and comparing EPSCs from PNs and PVs in patches and interpatches of the same tangential slice, showed that responses in patches were larger ( $p<0.001$, t-test) than in interpatches (Figure 5A, B, D, G), which correlated with the higher input density to $L 1$ patches (Figure $2 \mathrm{~B}$ ). Different from the $\mathrm{dLGN} \rightarrow \mathrm{V} 1$ input, feedback from LM to patches evoked similar ( $p=0.38$, Wilcoxon signed-rank test) size EPSCs from PVs $(529.5 \pm 305.9 \mathrm{pA}, \mathrm{n}=23)$ and PNs $(598.9 \pm 433.2 \mathrm{pA}, \mathrm{n}=23)$ (Figure $5 \mathrm{C}, \mathrm{G})$. The 
even strength of LM input to V1 PNs and PVs in patches was also observed in the equal spread of activation across the dendritic tree and the matching current densities (Figure 5F). The spatial extent and current density in LM-activated patch- was larger than in interpatch-PNs $(p<0.01$, two-sample t-test), whereas both measurements were similar for PVs (Figure 5F), suggesting that in the $\mathrm{LM} \rightarrow \mathrm{V} 1$ pathway the balanced activation in patches is due to stronger input to $\mathrm{PNs}$. In contrast, responses from PVs $(244 \pm 186.4$ pA, $n=23)$ in interpatches were 1.9-fold larger $(p<$ 0.001 , paired t-test) than from $P N s$ (130.1 $\pm 145.9 \mathrm{pA}, \mathrm{n}=23$ ) (Figures $5 E, G)$, an imbalance that was similar to that of dLGN inputs. Thus, balanced strength of input to PVs and PNs is a signature feature of higher order thalamic pathways, but not of top-down feedback, which is modulespecific.

\section{Long-range inputs to patches and interpatches drive distinct inhibitory subnetworks}

The spatial clustering in the cell-specific strength of activation by inputs to L1 raised the question whether inhibition is non-uniformly mapped across the sheet of $\mathrm{V} 1$.

Clustering of GABAergic neurons. It has been reported that PV- and SOM-expressing GABAergic neurons in L2/3 of mouse V1 are clustered in the tangential plane and are radially aligned in L5 with subcortically projecting PNs (Ebina et al., 2014. Maruoka et al., 2017). To test this notion directly we cut tangential sections through V1 of PVtdT mice and found a striking clustering of tdT labeled fibers in L1 with a center-to-center spacing of $\sim 120 \mu \mathrm{m}$ (Figure S6). Clustering of PVtdT fibers was also present in surrounding cortex, most notably in $L M, A L$ and primary auditory cortex (AUDp). To determine whether M2 and PVs are spatially registered, we stained tangential sections through $\mathrm{V} 1$ of PVtdT mice with an antibody against M2. We found that processes of PVs in L1-2/3 were strikingly clustered (Figure 6B-K). In the outer half of L1 (L1A, Figure $3 \mathrm{~A}$ ) the clusters contained mostly thick, smooth dendrites which overlapped with $\mathrm{M} 2+$ patches (Figure 6B-D). In contrast, in L1B and the top of L2/3 the PVtdT clusters switched to overlap with M2- interpatches. In L1B PVtdT clusters contained beaded dendrites and axons to which cell bodies and pericellular baskets, characteristic for basket cells (BC), were added in L2 (Figure 6A, E-K). The complementary mapping schemes were confirmed by the shifting distributions in PVtdT fluorescence intensities from patches $\left(p>10^{-15}, \mathrm{KS}\right)$ to interpatches $(p=$ $3.42 \times 10^{-4}, \mathrm{KS}$ ) across the depth of L1-2 (Figures $\left.6 \mathrm{~L}, \mathrm{M}\right)$. In separate counts we found that the PV cell density in the top $160 \mu \mathrm{m}$ of L2/3 was $37 \%$ higher in interpatches $\left(204 / \mathrm{mm}^{2}\right)$ than in patches $\left(128 / \mathrm{mm}^{2}\right)$. To further support the module-selective mapping of PV axons we filled $8 \mathrm{PVs}$ with biocytin. We found that in both patches $(\mathrm{N}=3)$ and interpatches $(\mathrm{N}=5)$ axons were largely confined to a $\sim 100 \mu \mathrm{m}$-wide cluster and projections to the surround were sparse (Figure $6 \mathrm{~N}$ ). 
Notably, this was the case also for the projections parallel to the long axis of an obliquely cut, oval interpatch.

Next, we asked whether the clustering of GABAergic neurons is subtype-specific. Most (>97\%) types of GABAergic neurons express the vesicular GABA transporter (VGAT) (Uematsu et al. 2008). We therefore used VGAT-EYFP mice to look for patchy VGAT expression in V1. We found that VGAT expression in L1-2 was non-uniform but less discrete than the clustered projections from the dLGN (Figure S7A-F). Nevertheless, compared to unity of the shuffled patch/interpatch ratio, VGAT fluorescence intensity was significantly $(p<0.035)$ higher in interpatches, consistent with the clustering of PV (Figure 6M).

The phase shift of PVtdT and M2 between outer and inner parts of L1 suggested that this might be due to the differential distributions of chandelier $(\mathrm{ChC})$ and $B C$ dendrites in $L 1 A$ and $1 B$ relative to patches and interpatches (Figure 6A), similar to those found in mouse prefrontal cortex (Miyamae et al., 2017). To approach this question we compared the density of PVtdT boutons in cartridges apposed to Ankyrin G immunolabeled axon initial segments (AIS) (Blasquez-Lorca et al., 2015) in $75 \mu \mathrm{m}$-wide L2/3 'columns' aligned with M2+ patches and M2- interpatches of PVtdT mice (Figure S8A-D). For analysis we selected radially oriented, tapered AISs of putative PNs. Consistent with the higher density of interneurons (Figure 6L) we found that the Ankyrin G-positive AIS density in interpatches was $21 \%$ lower ( $p<0.01$, paired t-test) than in patches (Figure S8E), suggesting that the overall cell density across the cortical sheet is constant. Most notably we found that the length density of PV boutons in cartridges was $52 \%$ higher $(p<0.01$, paired t-test) in patches, which supports the notion that ChCs connections are preferentially aligned with patches (Figure 6A; S7E).

Subnetwork-specific FFI. Because FFI onto PNs depends on the strengths of excitatory longrange input to PVs and their inhibitory output (Isaacson and Scanziani, 2011), we performed whole cell recordings of unitary uEPSCs and uIPSCs from synaptically connected pairs of L2/3 PVs and PNs in V1 of PVtdT mice. To distinguish patches from interpatches we traced dLGN input to L1 with AAV2/9.CAG.ChR2.Venus. We first identified PVs by the expression of tdT and their non-adapting fast spiking properties and then searched for a neighboring PNs based on the triangular morphology and the regular spiking pattern (Figure 7A-C). Recordings from PNs were performed at holding potentials of $-70 \mathrm{mV}$ with pipettes containing a high [Cl]. This enhanced the inward-directed (Luo et al., 2013), monosynaptic uIPSCs (Dong et al., 2004) (latency $2.4 \pm 0.3$ 
ms [patches $n=22$ ], $2.3 \pm 0.3 \mathrm{~ms}$ [interpatches, $n=26$ ]), elicited by spikes (recorded in current clamp) from presynaptic PVs (Figure 7D, E). We found that uIPSCs and charge transfer (averaged across 50-150 repetitions) from PNs in interpatches (209 \pm 149 pA, $2.79 \pm 1.64$ pC, $\mathrm{n}=22$ pairs) were 5-7-fold larger ( $p<0.001$, two-sample t-test) than in patches (30 $\pm 15 \mathrm{pA}, 0.54$ $\pm 0.29 \mathrm{pC}, \mathrm{n}=26$ pairs) (Figure 7F, G). Bath-application of Picrotoxin $(50 \mu \mathrm{M})$ completely abolished uIPSCs in patches and interpatches (Figure 7D-F), demonstrating that responses were mediated via $\mathrm{GABA}_{\mathrm{A}}$-receptors. Although $\mathrm{PVs}$ were less numerous in patches than interpatches, the probability of PVs $\rightarrow$ PNs contacts in patches (72\%, 26 PNs/36 PVs) and interpatches (88\%, 22 PNs/25 PVs) were similar (Figure $7 \mathrm{H})$. These results show that the FFI subnetworks in patches and interpatches are not identical.

Subnetwork-specific E/I balance. Subnetwork-specific FFI differentially affect the mono- and polysynaptic excitation of PNs, which project back onto PVs and in turn may provide distinct feedback inhibition to PNs in patches and interpatches (Isaacson and Scanziani, 2011). In experiments separate from those shown in Figure 7, we measured the strength of uEPSCs and uIPSCs from reciprocally connected $P N \leftrightarrow P V$-pairs in patches and interpatches. The experimental procedures were similar to those in Figure 7, except that we also measured uEPSCs from PVs elicited by spikes in presynaptic PNs (Figure 8A-D). We found that uEPSC amplitudes and charge transfers from $\mathrm{PVs}$ in patches $(42.2 \pm 9.2 \mathrm{pA})$ and interpatches $(29.1 \pm 4.7 \mathrm{pA})$ were similar $(p=$ ns, two-sample t-test) (Figure 8C-F, G, I). In sharp contrast, mean uIPSCs and uIPSQs recorded from $\mathrm{PNs}$ in interpatches $(238.5 \pm 45.9 \mathrm{pA}, \mathrm{n}=12$ pairs) were 8.8-fold larger $(p<0.001$, twosample t-test) than in patches $(27 \pm 4.5 \mathrm{pA}(\mathrm{n}=12$ pairs) (Figure $8 \mathrm{C}-\mathrm{F}, \mathrm{H}, \mathrm{J})$. Bath application of Picrotoxin $(50 \mu \mathrm{M})$ completely blocked uIPSCs in patches (10/10) and interpatches (11/11) (Figures $8 \mathrm{C}, \mathrm{D})$. In the returning excitatory connections, bath application of DNQX $(20 \mu \mathrm{M})$ completely blocked uEPSCs in patches (9/9) and interpatches (10/10), indicating that uEPSCs were mediated via AMPA receptors (Figures $8 \mathrm{C}, \mathrm{D}$ ). To estimate the relative strength of $\mathrm{E}$ and I we determined uIPSCS/UEPSCs ratios in patches and interpatches. In patches I/E was $0.89 \pm$ 0.21 ( $n=12$ pairs) and differed significantly ( $p<0.001$, two-sample t-test) from $10.9 \pm 2.5(\mathrm{n}=12$ pairs) in interpatches (Figure $8 \mathrm{H}$ ). Similar $\mathrm{l} / \mathrm{E}$ ratios were obtained for synaptic charge transfer (Figure 8J).

In both patches and interpatches uIPSCs lagged uEPSCs by $2.21 \pm 0.14 \mathrm{~ms}$ and $2.35 \pm 0.16 \mathrm{~ms}$, respectively (Figure $8 \mathrm{~L}$ ). Importantly, uIPSCs in interpatches showed significantly faster rise times ( $p<0.001$, two-sample t-test) than in patches (Figure 8M). The results show that although 
synaptic activation of PVs tracked that of PNs, the opposing inhibition of PNs is markedly stronger and faster in interpatches than in patches.

Because of the low density of PVs in patches, reciprocally connected PV $\leftrightarrow$ PNs pairs (> $50 \mu \mathrm{m}$ apart) were less common than in interpatches. Despite this difference the probability of mutually connected pairs in patches (81\%) and interpatches (86\%) was high (Figure 8K) and similar to $\mathrm{PV} \rightarrow \mathrm{PN}$ pairs (Figure $7 \mathrm{H}$ ). In contrast only $33 \% \mathrm{PV} \leftrightarrow \mathrm{PNs}$ connections within a $>50 \mu \mathrm{m}$ radius crossed the patch/interpatch border (Figure $8 \mathrm{~K}$ ). These findings are consistent with results showing that PVs are integrated into different subnetworks of PNs with similar response properties (Znamenskiy et al., 2018).

\section{DISCUSSION}

We have found two interdigitating maps of $\mathrm{M} 2+$ patches and M2- interpatches in L1 of mouse V1 and show that PV-mediated inhibition of neighboring L2/3 PNs is significantly stronger in interpatches than in patches. We further show that each network is driven by distinct long-range inputs to dendrites in $L 1$. While patches are the preferred targets of $d L G N, L M$ and $A L$, interpatches receive inputs from the LP thalamus and PM. Although these inputs are modulespecific the strength of synaptic activation of PVs and PNs in patches and interpatches is pathway-specific. Specifically, in patches dLGN input excites PVs more strongly than PNs, whereas both cells types are activated equally by feedback from LM. Similarly, in interpatches LM inputs to PVs and PNs are matched whereas LM input excites PVs more strongly. Together the results show that long-range inputs play a role in the $E / I$ balance but suggest that the spike output of PNs is filtered by the activation threshold of PVs and their diverse strengths of inhibiting PNs in patches and interpatches. Although the efficacy of E/I coupling is thought to provide for modulespecific tuning (Isaacson and Scanziani, 2011), the net impact on the output from patches and interpatches is shortening the integration window and exacting the selection of synchronous inputs (Gabernet et al., 2005). However, the opposing overall inhibition is strongest in interpatches, reducing response gain, increasing stimulus sensitivity (Atallah et al., 2012; Katzner et al., 2011) and improving the robustness of temporal frequency tuning in the interpatch module (Ji et al., 2015; Zhu et al., 2015).

\section{Patchy networks in L1 of V1}

Finding interdigitating maps of 60-80 $\mu \mathrm{m}$-wide clusters of thalamic and intracortical inputs in L1 was unexpected, given the salt-and-pepper organization of mouse V1 (Ohki and Reid, 2007). 
However, interdigitating microcolumns of subcortically and intracortically projecting L5 neurons, including clusters of L2/3 neurons and bundled apical dendrites with similar orientation and SF tuning, were recently found in mouse V1 (Kondo et al., 2016; Maruoka et al., 2017R; Ringach et al., 2016). It was puzzling, though, why L5 neurons were mapped hexagonally with a periodicity of 30-45 $\mu \mathrm{m}$ and showed an organization that differed from the quasi-rectangular lattice and the $120 \mu \mathrm{m}$ periodicity of M2 patches we have found in L1 (Ji et al., 2015). In speculating about the alignment of infra- and supragranular maps we noted in tangential sections that upper layers occupy a $\sim 25 \%$ larger area than infragranular layers (A. Burkhalter, unpublished results). Thus, to maintain topographic alignment across layers, ascending dendrites from L5 may be bundled proximally (Innocenti and Vercelli, 2010), form wider tufts distally and become targets of thalamocortical and intracortical connections in L1-2.

Clustered long-range projections to $L 1$ are known from horizontal and feedback networks in primate, cat and mouse V1 (Ji et al., 2015; Martin and Whitteridge, 1984; Stettler et al., 2002). Here, we show that L1 projections from dLGN and the LP are also clustered in interdigitating maps. Different from the canonical core $d L G N \rightarrow V 1$ pathway to L3-4 (Bickford et al., 2015), input to $L 1$ originates in the dLGN shell. The shell receives connections from direction-selective retinal ganglion cells and the superior colliculus and without prior cortical processing delivers orientation and direction selective signals (Bickford et al., 2015; Cruz-Martin et al. 2014; Roth et al. 2016) to patches in L1. Recordings from thalamocortical terminals have shown that the dLGN shell adds locomotion and saccade signals to visual responses and informs dendrites in L1 whether the speed of self-motion is matched to the visual flow of the environment (Roth et al., 2016). LP input to $L 1$ derives from multiple subnuclei (Bennett et al., 2019), including the anterior portion whose projections we have traced most successfully. Similar to the dLGN shell, LP receives direction selective input from the retina and the superior colliculus (Allen et al., 2016; Zhou et al., 2017) but unlike dLGN shell projections, LP inputs to L1 are tuned to the mismatch of self-motion and visual flow and provide information about moving objects in the environment (Roth et al., 2016).

We found that intracortical feedback connections are clustered as well, but patch- and interpatchprojections are not cleanly sorted by their sources in the ventral or dorsal streams (Wang et al., 2012). Instead patch-projections originate from ventral- (i.e. LM) and dorsal-stream areas (i.e. $\mathrm{AL}$ ), whereas another dorsal area, $\mathrm{PM}$, preferentially targets the boundary region shared by patches and interpatches. This diversity suggests that the dorsal network branches into ALdominated and PM-dominated sub-streams, perhaps similar to those in the primate occipito- 
parietal network specialized for visually guided actions and spatial navigation (Kravitz et al., 2011). The convergence of feedback from $L M$ and $A L$ suggests that patches multiplex inputs (Kampa et al., 2011) from functionally non-matching presynaptic neurons (Glickfeld et al., 2013; Marshel et al., 2012). Alternatively, responses of feedforward and feedback terminals may differ, suggesting that feedforward signals are transformed in higher areas, then returned for subtraction from bottom-up inputs to $\mathrm{V} 1$, which sends the error message back up the hierarchy to optimize sensory predictions (Zmarz and Keller, 2016).

\section{Subnetwork-selective targeting of PNs and PVs}

The overlap of clustered thalamocortical and cortico-cortical projections to patches and interpatches suggests that the inputs target L1 dendrites of PNs and PVs whose cell bodies map to distinct $X / Y$ coordinates in the layers below (Cruikshank et al., 2012; Johnson and Burkhalter, 1996; Miyamae et al., 2017). Recordings of EPSCs from L2/3 PNs and PVs elicited by dLGN and LM inputs support this interpretation, and in agreement with spatial clustering of connections, show that patch-inputs are stronger than interpatch-inputs. LP inputs to PNs show a similar structure-function relationship. Similar to the activation of cortical feedforward pathways (D'Souza e al., 2016; Yang et al., 2013) dLGN inputs to PVs were stronger than to PNs. This differed from the balanced LP and LM inputs to PVs and PNs, which is typical for intracortical feedback connections (D'Souza et al., 2016).

Synaptic inputs to PN and PV dendrites in L1 readily elicit spikes from cell bodies in the layers below (Cauller and Connors, 1994; Hu et al., 2010; Larkum et al., 2009). Our results show that the absolute and relative strengths of long-range inputs to PNs and PVs vary by pathway, patches $(P V / P N \approx 2: 1)$ and interpatches (PV/PN $\approx 1: 1$ ), and demonstrate that the balance by which the network-stabilizing FFI tracks excitation (Xue et al, 2014) is pathway- and module-specific. Strong $\mathrm{FFI}$ in the dLGN $\rightarrow \mathrm{V} 1$ pathway may select for synchronous inputs and enhance stimulus detection, whereas weaker $\mathrm{FFI}$ in the $\mathrm{LP} \rightarrow \mathrm{V} 1$ and $\mathrm{LM} \rightarrow \mathrm{V} 1$ pathways may broaden the integration window of convergent inputs and enhance the discriminability of stimulus features (Gabernet, 2005; Wang et al., 2010). Although FFI in patches and interpatches is proportional to the long-range input and the tuning properties of PNs and PVs (Hofer et al., 2011) the inhibitory effect of this input on PN spike output is stronger in patches than interpatches.

Consistent with the nonrandom connectivity in $L 2 / 3$, but counter to areal uniformity (Kim et al., 2017), we have found that PV somata and terminals in L1-2 are spatially clustered (Ichinhoe et 
al. 2003; Maruoka et al., 2017; Znamenskiy et al., 2018). Similar to the spatial clustering of IPSC amplitude (Ebina et al., 2014) and consistent with clustering of visual response properties and strong inhibition between visually co-tuned PVs and PNs (Ji et al., 2015; Znamenskiy et al., 2018) the results show stronger local inhibition in interpatches than in patches. This may be due to larger, more proximal and/or the higher density of PV synapses onto PNs (Kubota et al., 2015; Stüber et al., 2015). Although the subcellular organization remains speculative, our results show that independent of the mode of activation by long-range inputs spikes from PVs in interpatches evoke at least 5-fold larger uIPSCs in interpatches than in patches. This strong locally generated inhibition may lower the gain of PN spike output and increase the sensitivity and robustness of responses to TF in interpatches (Atallah et al., 2012; Ji et al., 2015; Katzner et al., 2011; Zhu et al. 2015).

A possible role of the patch/interpatch organization is the selective tuning of visual responses of projection neurons. The most direct link comes from L5 PNs. Here, subcortically (i.e. pons, LP, superior colliculus, striatum) projecting L5B PNs overlap with PV clusters suggesting that they receive interpatch input onto thick dendrites in L1 (Kim et al., 2015; Maruoka et al. 2017). These cells are sensitive to high TF and low SF, properties we have found in L2/3 of interpatches (Ji et al. 2015; Kim et al., 2015). In contrast, L5A intracortically projecting (i.e. local V1, medial and lateral extrastriate visual areas) neurons which are excluded from $\mathrm{PV}$ clusters may receive patch input onto thin dendrites in L1 (Kim et al., 2015; Maruoka et al, 2017). The subset of Efr3a-Cre L5A neurons are sensitive to high SF which is the preferred property of L2/3 patch neurons (Ji et al., 2015; Kim et al., 2015). The relationship with patches and interpatches of the L2/3 PNs we have studied remains to be determined. Among the many types of L2/3 V1 neurons (Harris et al., 2018) few have dedicated projection targets and most are of the broadcasting type, projecting to multiple functionally distinct areas (Andermann et al., 2011; Han et al., 2018; Marshel et al., 2011) making a clean separation into patch and interpatch L2/3 neurons unlikely.

Besides the overlap of PV-positive BC dendrites with interpatches in the inner part of L1, different PV-dendrites reach the pial surface and preferentially branch in patches. The radial distribution of genetically labeled interneuron dendrites across L1 suggests that these processes belong to ChCs with cell bodies in L2/3 (Taniguchi et al, 2013; Tasic et al., 2018). The clustering of putative $\mathrm{ChC}$ dendrites is consistent with reports that AIS of cortico-cortically projecting L2/3 PNs are innervated in $\sim 60 \mu \mathrm{m}$ wide clusters (Blazquez-Llorca et al., 2015; Farinas and DeFelipe, 1991). Our findings confirm these results and show that the innervation density of L2/3 AIS in patches is 
higher than in interpatches, the opposite of what we have found in the projection density of PVs. $\mathrm{L} 1$ patches receive dense inputs from the dLGN, LM and $A L$, which when stimulated may recruit ChC-mediated FFI in layer 2/3 PNs (Woodruff et al., 2011). Unlike BC-mediated FFI, which regulates spike output from L2/3 PN by coordinating the dendritic integration of bottom-up and top-down inputs (Larkum et al. 2007; Larkum, 2013), ChC-mediated FFI by L1 input may suppress PN output at the AIS and cancel the error signal to higher visual areas for example during eye movements.

\section{STAR * METHODS}

Detailed methods are provided in the online version of this paper and include the following:

- EXPERIMENTAL MODEL AND SUBJECT DETAILS

Animals

- METHOD DETAILS

Tracing of connections

Immunostaining

Slice preparation

Subcellular Channelrhodopsin-2 assisted circuit mapping (sCRACM)

Photostimulation

Recordings from synaptically connected pairs

- DATA ANALYSIS

Contour plots of patches and interpatches

Quantification of florescence intensity

EPSCs and IPSCs

Confocal imaging and neuron reconstruction

Statistics

\section{SUPPLEMENTAL INFORMATION}

Supplemental information includes Supplemental Experimental Procedures and eight figures.

\section{AUTHOR CONTRIBUTIONS}

P.B., R.D.D and A.B. designed the research. P.B. and R.D.D. performed the physiological and most of the anatomical experiments, with significant help from A.M., W.J. and A.B. All authors 
contributed to the data analysis. A.M implemented software. A.B., P.B. and R.D.D. wrote the paper with inputs from all authors.

\section{ACKNOWLEDGEMENTS}

We thank Hongkui Zheng of the Allen Institute for Brain Science for the AAV2/1hSyn.tdTomato.WPRE.bGH, James Fitzpatrick and Dennis Oakley (Washington University Center for Cellular Imaging) and Katia Valkova for technical support. Thanks also to Tim Holy for the VGAT-ChR2-EYFP mice. Supported by National Eye Institute grants RO1 EY16184, RO1 EY022090, RO1 EY027383 and the McDonnell Center for Systems Neuroscience.

\section{DECLARTION OF INTERESTS}

The authors declare no competing interests. 


\section{REFERENCES}

Agrawal, H.C., Davis, J.M., and Himwich, W.A. (1968). Developmental changes in mouse brain: wight, water content and free amino acids. J. Neurochem. 15, 917-923.

Allen, A.E., Procyk, C.A. Howarth, M., Walmsley, L., and Brown, T.M. (2016). Visual input to the mouse lateral posterior and posterior thalamic nuclei: photoreceptive origins and retinotopic order. J. Physiol. 594, 1911-1929.

Andermann, M.L., Kerlin, A.M., Roumis, D.K., Glickfeld, L.L., and Reid, R.C. (2011). Functional specialization of mouse higher visual cortical areas. Neuron 72, 1025-1039.

Atallah, B.V., Burns, W., Carandini, M., and Scanziani, M. (2012). Parvalbumin-expressing interneurons linearly transform cortical responses to visual stimuli. Neuron 73, 159-170.

Ayzenshtat I., Karnani, M.M., Jackson, J., and Yuste, R. (2016). Cortical control of spatial resolution by VIP+ interneurons. J. Neurosci. 36, 11498-11509.

Bennett, C., Gale, S.D., Garret, ME., Newton, M.L., Callaway, E.M.., Murphy, G.J., and Olsen, S.R. (2019). Higher-order thalamic circuits channel parallel streams of visual information in mice. Neuron Feb. 27. (Epub ahead of print).

Bickford, M.E., Zhou, N., Krahe, T.E., Govindaiah, G., and Guido, W. (2015). Retinal and tectal "driver-like" inputs converge in the shell of the mouse dorsal lateral geniculate nucleus. J. Neurosci. 35, 10523-10534.

Blazquez-Llorca, L., Woodruff, A., Inan, M., Anderson, S.A., Yuste, R., DeFelipe, J., and MerchanPerez, A. (2015). Spatial distribution of neurons innervated by chandelier cells. Brain Struct. Funct. 220, 2817-2834.

Cauller, L.J., and Connors, B.W. (1994). Synaptic physiology of horizontal afferents to layer 1 in slices of rat $\mathrm{S} 1$ neocortex. J. Neurosci. 14, 751-762.

Cruikshank, S.J., Ahmed, O.J., Stevens, T.R., Patrick, S.L., Gonzalez, A.M., Elmaleh, M., and Connors, B. W. (2012). Thalamic control of layer 1 circuits in prefrontal cortex. J. Neurosci. 32, 17813-17823.

Cruz-Martín, A., El-Danaf, R.N., Osakada, F., Sriram, B., Dhande, O.S., Nguyen, P.L., Callaway, E. M., Gosh, A., and Huberman, A.D. (2014). A dedicated circuit links direction-selective retinal ganglion cells to the primary visual cortex. Nature 507, 358-507.

DaCosta, N. M., and Martin, K.A.C. (2013). Sparse reconstruction of brain circuits: Or, how to survive without a microscopic connectome. Neuroimage 80, 27-36.

Dong, H., Shao, Z., Nerbonne, J.M., and Burkhalter, A. (2004).Differential depression of inhibitory synaptic responses in feedforward and feedback circuits between different areas of mouse visual cortex. J. Comp. Neurol. 475, 361-373. 
D'Souza, R. D., Meier, A.M., Bista, P., Wang, Q., and Burkhalter, A. (2016). Recruitment of inhibition and excitation across mouse visual cortex depends on the hierarchy of interconnecting areas. eLife 2016; 5:e193332, DOI:10.7554/eLife.19332.

Ebina,T., Sohya, K., Imayoshi, I., Yin,S-T., Kimura, R., Yanagawa, Y., Kameda, K., Hioki, H., Kaneko, T., and Tsumoto, T. (2014). 3D clustering of GABAergic neurons enhances inhibitory actions on excitatory neurons in the mouse visual cortex. Cell Report 9, 1-12.

Farinas, I., and DeFelipe, J. (1991). Patterns of synaptic input on cortico-cortical and corticothalamic cells in cat visual cortex. II. The axon initial segment. J. Comp. Neurol. 304, 70-77.

Felleman, D.J. and Van Essen, D.C. (1991). Distributed hierarchical processing in the primate cerebral cortex. Cereb. Cortex, 1-47.

Gabernet, L., Jadhav, S.P., Feldman, D.E., Carandini, M., and Scanziani, M. (2005). Somatosensory integration controlled by dynamic thalamocortical feed-forward inhibition. Neuron 48, 315-327.

Glickfeld L.L., Andermann, M., Bonin, V., and Reid, R.C. (2013). Corticocortical projections in mouse visual cortex are functionally target-specific. Nat. Neurosci. 16, 219-226.

Gomeza, J., Shannon, H., Kostenis, E., Felder, C., Zhang, L., Brodkin, J., Grinberg, A., Sheng, H., and Wess, J. (1999). Prononced pahramcologic deficits in M2 muscarinic acetylcholine receptor kockout mice. Proc. Natl. Acad.Sci. USA. 96, 1692-1697.

Han, Y., Kebschull, J.M., Cambell, R.A.A., Cowan, D., Imhof, F., Zador, A.M., and Mrsic-Flogel, T.D. (2018). The logic of single-cell projection for visual cortex. Nature 556, 51-56.

Harris, J.A., Mihalas, S., Hirokawa, K.E., Whitsell, J.D., Knox, J., Bernard, A., Bohn, P., Caldejon, S., Casal, L., Cho, A., Feng, D., Gaudreault, N., Graddis, N., Groblewski, P.A., Henry, A., Ho, A., Howard, R., Kuan, L., Lecoq, J., Luviano, J., McConoghy, S., Mortrud, M.T., Naeemi, M., Ng, L., Oh, S.W., Ouellette, B., Sorensen, S.A., Wakeman, W., Wang, Q., Williford, A., Phillips J.W., Jones, A., Koch, C., and Zeng, H. (2018). The organization of intracortical connections by layer and cell class in the mouse brain. bioRxiv posted Apr. 1, 2018. Doi: http://dx.doi.org/10.1.1101/292961.

Hofer, B.B., Ko, H., Pichler, B., Vogelstein, J., Ros, H., Zeng, H., Lein, E., Lesica, N.A., MrsicFlogel, T.D. (2011). Differential connectivity and response dynamics of excitatory and inhibitory neurons in visual cortex. Nat. Neurosci. 14, 1045-1052.

Horvát, S., Gămănut, R., Ercsey-Ravaz, M., Magrou, L., Gămănut, B., Van Essen, D.C., Burkhalter, A., Knoblauch, K., Trorczkai,Z., and Kennedy, H. (2016). Spatial embedding and 
wiring cost constrain the functional layout of the cortical network of rodents and primates. PLOS Biol. DOI:10.1371/journal.pbio.1002512.

Hu, H., Martina, M., and Jonas, P. (2010). Dendritic mechanism underlying rapid synaptic activation of fast-spiking hippocampal interneurons. Science 327, 52-58.

Hubel, D.H., and Wiesel, T.N. (1962). Receptive fields, binocular interaction and functional architecture in the cat's visual cortex. J. Physiol. 160, 106-154.

Hubel, D.H., and Wiesel, T.N. (1974). Uniformity of monkey striate cortex: a parallel relationship between field size, scatter, and magnification factor. J. Comp. Neurol. 158, 295-305.

Ichinohe, N., Fujiyama, F., Kaneko, T., and Rockland, K.S. (2003). Honeycomb-like mosaic at the border of layers 1 and 2 in cerebral cortex. J. Neuroscience. 23, 1372-1382.

Innocenti, G.M., and Vercelli, A. (2010). Dendritic bundles, minocolumns, columns, and cortical output units. Front. Neuroanat. 4, 1-7. doi:10.3389/neuro.05.011.2010.

Isaacson, J.S., and Scanziani, M. (2011). How inhibition shapes cortical activity. Neuron 72, 231243.

Johnson, R.R., and Burkhalter, A. (1996). Microcircuitry of forward and feedback connections within rat visual cortex. J. Comp. Neurol. 368, 383-398.

Ji, W., Gămănut, R., Bista, P., D’Souza, R.D.D., Wang, Q., and Burkhalter, A. (2015). Modularity in the organization of mouse primary visual cortex. Neuron 87, 632-643.

Kampa, B.M., Roth, M.M., Göbel, W., and Helmchen, F. (2011). Representation of visual scenes by local neuronal populations in layer 2/3 of mouse visual cortex. Front. Neural Circuits 5, 18 Dec 12. Doi: 10.3389/fncir.2011.00018.

Karnani, M.M., Agetsuma, M., and Yuste, R. (2014). A blanket of inhibition: functional inferences from dense inhibitory connectivity. Curr. Opin. Neurobiol. 26, 96-102.

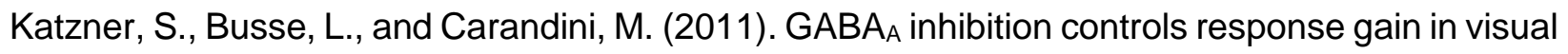
cortex. J. Neurosci. 31, 5931-5941.

Kim, E.J., Juavinett, A.L., Kyubwa, E.M., Jacobs, M.W., and Callaway, E.M. (2015). Three types of cortical layer 5 neurons that differ in brain-wide connectivity and function. Neuron 88, 12531267.

Kim, Y., Yang, G.R., Pradhan, K., Venkataraju, K.U., Bota,M., Garcia del Molino, L.C., Fitzgerald,G., Ram,K., He, M., Levine, J.M., Mitra, P., Huang, Z.J., Wang, X-J, and Osten, P. (2017). Brain-wide maps reveal stereotyped cell-type-based cortical architecture and subcortical sexual dimorphism. Cell 171, 456-469. 
Kondo, S., Yoshida, T., and Ohki, K. (2016). Mixed functional microarchitectures for orientation selectivity in mouse primary visual cortex. Nat. Commun. 7, 13210. doi: $10.1038 /$ ncomms 13210

Kravitz, D.J., and Saleem, K.S., Baker, C.I., and Mishkin, M. (2011). A new neural framework for visuospatial processing. Nat Rev. Neurosci. 12, 217-230.

Kubota, Y., Kondo, S., Nomura, M., Hatada, S., Yamaguchi, N., Mohamed, A.A., Karube, F., Lübke, J., and Kawaguchi, Y. (2015). Functional effects of distinctive innervation styles of pyramidal cells by fast spiking cortical interneurons. Elife 4, e07019: 1-27

Larkum, M.E., Waters, J., Sakmann, B., and Helmchen, F. (2007). Dendritic spikes in apical dendrites of neocortical layer 2/3 pyramidal neurons. J. Neurosci. 27, 8999-9008.

Larkum, M. E., Nevian, T., Sandler, M., Polsky, A., and Schiller, J. (2009). Synaptic integration in tuft dendrites of layer 5 pyramidal neuron: a new unifying principle. Science 325, 756-760.

Larkum, M.E. (2013). A cellular mechanism for cortical associations: an organizing principle for the cerebral cortex. Trends in Neurosci. 36, 141-151.

Lee, S-H., Kwan, A.C., Zhang, S., Phoumthipphavong, V., Flannery, J.G., Masmanidis, S.C., Taniguchi, H., Huang, Z.J., Zhang, F., Boyden, E.S., Deisseroth, K., and Dan, Y. (2012). Activation of specific interneurons improves $\mathrm{V} 1$ feature selectivity and visual perception. Nature 488, 379-383.

Lee, S-H., Kwan, A.C., and Dan, Y. (2014). Interneurons subtypes and orientation tuning. Nature 508, (7494):E1-2.DIO 10.1038/nature13128.

Li, L-Y., Ji, X-Y., Liang, F., Li, Y-T., Xiao, Z., Tao, H.W., and Zhang, L.I. (2014). A feedforward inhibitory circuit mediates lateral refinement of sensory representation in upper layer $2 / 3$ of mouse primary auditory cortex. J. Neurosci. 34, 13670-13683.

TaniguchiMao, T., Kusefoglu, D., Hooks, B.M., Huber, D., Petreanu, L., and Svoboda, K. (2011). Long-range neuronal circuits underlying the interaction between sensory and motor cortex. Neuron 72,111-123.

Marshel, J.H., Garrett, M.E., Nauhaus, J., and Callaway, E.M. (2011). Functional specialization of seven mouse visual cortical areas. Neuron 72, 1042-1054.

Martin, K.A.C. and Whitteridge, D. 1984. Form, function and intracortical projections of spiny neurons in the striate visual cortex of the cat. J. Physiol. 353, 463-504.

Maruoka, H., Nagakawa, N., Tsuruno, S. Sakai, S., Yoneda, T., and Hosoya, T. (2017). Lattice system of functionally district cell types in the neocortex. Science $258,610-615$. 
Miyamae, T., Chen, K., Lewis, D.A., and Gonzalez-Burgos, G. (2017). Distinct physiological maturation of parvalbumin-positive neuron subtypes in mouse prefrontal cortex. J. Neurosci. 37, 4883-4902.

Muñoz, W., and Rudy, B. (2014). Spatiotemporal specificity in cholinergic control of neocortical function. Curr. Opin. Neurobiol. 26, 149-160.

Natan, R., Rao, W., and Geffen, M.N. (2017). Cortical interneurons differentially shape frequency tuning following adaptation. Cell Reports 21, 878-890.

Ohki, K., Chung, S., Ch'ng, Y.H., Kara, P., and Reid, R.C. (2005). Functional imaging with cellular resolution reveals precise micro-architecture in visual cortex. Nature 433, 597-603.

Ohki, K., and Reid, R.C. (2007). Specificity and randomness in the visual cortex. Curr. Opin. Neurobiol. 17, 401-407.

Petreanu, L., Mao, T., Sternson, S.M., Svoboda, K. (2009). The subcellular organization of neocortical excitatory connections. Nature 457, 1142-1145

Ringach, D.L., Mineault, P.J., Tring, E., Olivas, N.D., Garcia-Junco-Clemente, P., and Trachtenberg, J.T. (2016). Spatial clustering of tuning in mouse primary visual cortex. Nat. Comm. 7, 12270

Roth, M. M., Dahmen, J. C., Muir, D.R., Imhof F., Martini, F. J., and Hofer, S.B. (2016). Thalamic nuclei convey diverse contextual information to layer 1 of visual cortex. Nat Neurosci. 19, 299-307.

Sincich, L. C., and Horton, J. C. (2005). Input to V2 thin stripes arises from V1 cytochrome oxidase patches. J. Neurosci. 25, 10087-10093.

Stettler, D. D., Das, A., Bennett, J., and Gilbert, C. (2002). Lateral connectivity and contextual interactions in macaque primary visual cortex. Neuron 36, 739-750.

Strüber, M., Jonas, P., and Bartos, M. (2015). Strength and duration of perisomatic GABAergic inhibition depend on distance between synaptically connected cells. Proc. Natl. Acad. Sci. USA 112, 1220-1225.

Suter, B.A., O'Connor, T., Iyer, V., Petreanu, L.T., Hooks, B.M., Kiritani, T., Svoboda, K., and Shepherd, G.M. (2010). Ephus: multipurpose data acquisition software for neuroscience experiments. Front. Neural Circuits 4, 100.

Tasic, B., Yao, Z., Graybuck, L.T., Smith, K.A., Nguyen, T.N., Bertagnolli, D., Goldy, J., Garren, E., Economo, M.N., Viswanathan, S., Penn, O., Bakken, T., Menon, V., Miller, J., Fong, O., Hirokawa, K.E., Lathia, K., Rimorin, C., Tieu, M., Larsen, R., Casper, T., Barkan, E., Kroll ,M., Parry, S., Shapovalova, N.,V., Hischstein, D., Pendergraft, J., Sullivan, H.A., Lim, T.K., Szafer, A., Dee, N., Groblewski, P., Wickersham, I., Cetin, A., Harris, J.A., Levi, B.P., 
Sunkin,S.M., Madisen, L., Daigle, T.I., Looger, L., Bernard, A., Phillips, J., Lein, E., Hawrylycz, M., Svoboda, K., Jones, A.R., Koch, C., and Zeng, H. (2018). Shared and distinct trasnscriptomic cell types across neocortical areas. Nature 563,79-84.

Taniguchi, H., Lu, J., and Huang, Z.J. (2013). The spatial and temporal origin of chandelier cells in mouse neocortex. Science 339, 70-74.

Uematsu, M., Hirai, Y., Karube, F., Ebihara, S., Kato, A., Obata, K., Yoshida, S., Hirabayashi, M., Yanagawa, Y., and Kawaguchi, Y. (2008). Quantitative chemical composition of cortical GABAergic neurons revelaed in transgenic Venus-expressing rats. Cereb. Cortex 18, 15-30. Wang, Q., Sporns, O., and Burkhalter, A. (2012). Network analysis of cortico-cortical connections reveals ventral and dorsal processing streams in mouse visual cortex. J. Neurosci. 32, 43864399.

Woodruff, A.R., McGarry, L.M., Vogels, T.P., Inan, M., Anderson, S.A. and Yuste, R. (2011). State-dependent function of neocortical chandelier cells. J Neurosci. 31, 17872-17886.

Xue, M., Atallah, B.V., and Scanziani, M. (2014). Equalizing excitation-inhibition ratio across visual cortical neurons. Nature 511, 596-600.

Yang, W., Carrasquillo, Y., Hooks, B. M., Nerbonne, J.M. and Burkhalter, A. (2013). Distinct balance of excitation and inhibition in an interareal feedforward and feedback circuit of mouse visual cortex. J. Neurosci. 33, 17373-17384.

Zhou, N., Maire, P.S., Masterson, S.P., and Bickford, M.E. (2017). The mouse pulvinar nucelus: Organization of the tectorecipient zones. Vis. Neurosci. 34, E011.

Zhou, N., Masterson, S.P., Damron, J.K., Guido, W., and Bickford, M.E. (2018). The mouse pulvinar nucelus links the lateral extrastriate cortex, striatum, and amygdala. J. Neurosci. 38, 347-362.

Zhu, Y., Qiao, W., Liu, K., Zhong, H., and Yao H. (2015). Control of response reliability by parvalbumin-expressing interneurons in visual cortex. Nat. Comm. 6, 6802. dio: 10.1038/ncomms7802.

Zmarz, P. and Keller, G.B. (2016). Mismatch receptive fields in mouse visual cortex. Neuron 92, 766-772.

Znamenskiy, P, Kim, M-H, Muir, D.R., lacaruso, F., Hofer, S.B., and Mrsic-Flogel, T.D. (2018). Functional selectivity and specific connectivity of inhibitory neurons in primary visual cortex. bioRxiv Apr 4, 2018; dio:hhtp://dx.doi.org/10.1101/294835. 


\section{FIGURE LEGENDS}

\section{Figure 1. Anterograde viral tracing of dLGN and LP projections to L1 of V1.}

(A-C, E-G, I-L) Tangential sections through L1 showing M2+ patches and M2- interpatches in V1 of Chrm2tdT (A-C, E-G) and C57BL/6J mice (I-L). (A-C) Clustered dLGN projections traced with AAV2/1.hSyn.EGFP (green) overlap with M2tdT patches (purple). (D) Fluorescence intensity of dLGN input to patches (normalized to patches) is higher than to interpatches. (E-G) Clustered LP projections traced with AAV2/1.hSyn.EGFP (green) overlap with M2- interpatches. $(H)$ Fluorescence intensity of LP inputs to interpatches (normalized to patches) is higher than to patches. (I-L) Interdigitating projections from LP to interpatches traced with AAV2/1hSyn.tdTomato.WPRE.bGH (purple) and dLGN traced with AAV2/1.hSyn.EGFP (green) to patches. (M) Dense LP projections to interpatches where dLGN input is weak. Mean $\pm S D, K S$ test $(D, H, M)$.

\section{Figure 2. Anterograde viral tracing of LM and PM projections to L1 of V1.}

(A, C-E, F, G) Tangential sections through L1 of V1 of C57BL/6J (A, I, K) and Chrm2tdT mice (CE). (A) Overlapping clusters of dLGN projections traced with AAV2/1.hSyn.EGFP (green) and LM input traced by injection [ $\left.{ }^{\star}\right]$ of AAV2/1hSyn.tdTomato.WPRE.bGH (purple) to L1 of V1. (B) Fluorescence intensity of LM inputs to interpatches (normalized to patches) is weaker than to patches (M2+, Figure 1A-C). (C-E) Nonuniform distribution of inputs from PM traced with AAV2/1.hSyn.EGFP (green) partially overlapping with $\mathrm{M} 2+$ patches (purple) and M2interpatches. (F, G) Contour maps of M2tdT intensity. White boundaries indicate top (peaks of patches) and bottom (peaks of interpatches) quantiles. Cyan boundaries outline the middle two quantiles. (G) PM inputs to V1 (gold) fall preferentially into zone between top and bottom quantiles. $(H)$ Intensity profile shows that PM inputs to V1 overlap with patches and interpatches. Mean \pm SD. KS test. (I, K) Simultaneous tracing of PM (traced with AAV2/1hSyn.tdTomato.WPRE.bGH, purple) and AL (traced with AAV2/1.hSyn.EGFP, green) inputs to V1 superimposed onto immunolabeling for M2 (blue). At low magnification AL and PM projections show an interdigitating pattern. (L) Analysis of projection densities (boxed area in K) at high magnification shows that PM inputs (gold) involve patches (white outlines top 2 quantiles) and interpatches (cyan outlines bottom 2 quantiles). While AL projections avoid interpatches (Ji et al., 2015), note that $A L$ and PM projections to patches show an interdigitating pattern. 
Figure 3. Tangential slices: sCRACM-mapping of $d L G N \rightarrow V 1$ input to L1 onto L2/3 PNs and PVs in patches and interpatches. (A) Z-stack showing ChR2-Venus labeled dLGN $\rightarrow$ V1 projections in L1 and Alexa 594 hydrazide-filled pairs of L2/3 PNs and PVs in patch and interpatch. (B-E) Recordings from PN (black triangle) and PV (red circle) in patches and interpatches obtained in the same slice. (Bi, Biii) Each trace represents average of $\mathrm{EPSC}_{\mathrm{SCRACM}}(\mathrm{N}=3)$ to laser stimulation (blue dots, $75 \times 75 \mu \mathrm{m}$ grid) of ChR2-expressing dLGN $\rightarrow \mathrm{V} 1$ terminals. (Bii, Biv). Heatmaps of responses evoked at different locations of the dendritic arbor (Alexa 594 hydrazidefilled white profiles) of PN and PV shown in (Bi, Biii). (C) Each dot represents relative strength of

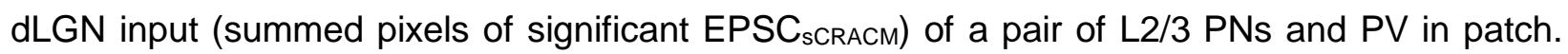
Red line: mean slope from zero. Blue line: mean slope after normalizing currents to mean conductance. (A, Di-iv, E) Recordings from PNs and PVs in interpatch. Same conventions as in $(B, C)$. (F) Distribution of dLGN input strength across dendritic arbor. Grey bars (number of pixels with non-zero EPSCs), PNs and PVs in patches and interpatches. Red bars, EPSC density (pA/ $\mu \mathrm{m}^{2}$ per $75 \mu \mathrm{m} \times 75 \mu \mathrm{m}$ pixel) in PVs and PNs of patches and interpatches. (G) Mean (dot) strength of $d L G N \rightarrow V 1$ EPSCs from PVs and PNs in patches and interpatches. (C, E, F, G) KS test. $\left({ }^{* * *} p<0.001,{ }^{* *} p<0.01\right)$.

Figure 4. Coronal slices: sCRACM-mapping of $d L G N \rightarrow V 1$ and $L P \rightarrow V 1$ input to L2/3

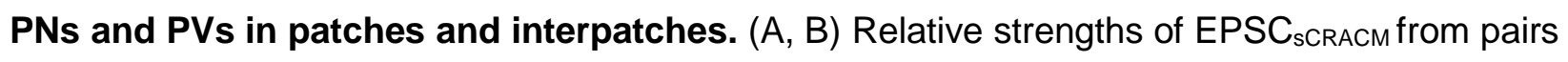
(dots) of L2/3 PNs and PVs evoked by $d L G N \rightarrow V 1$ input. Recordings from patches $(A)$ and interpatches (B) obtained in different slices. Mean slope of currents from zero (red), slope after

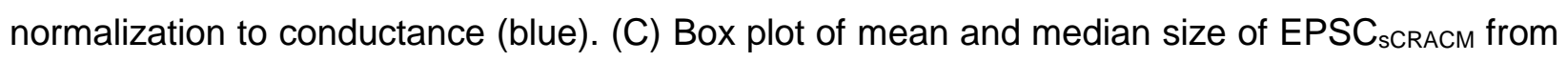

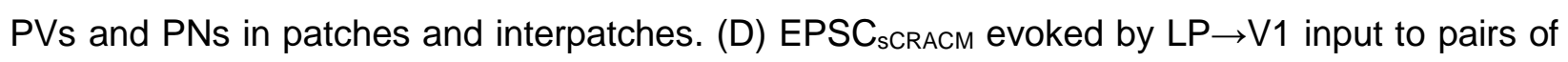

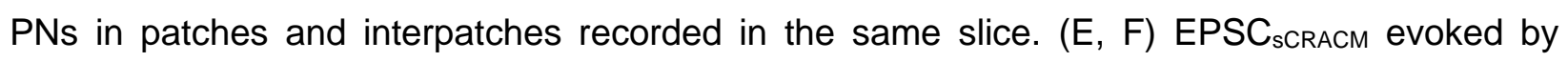
$\mathrm{LP} \rightarrow \mathrm{V} 1$ inputs to pairs of PNs and PVs in patches and interpatches. Different pairs recorded in different slices. $(\mathrm{G})$ Box plot EPSC $_{P V} /$ EPSC $_{P N}$ ratio in patches and interpatches. $(\mathrm{H})$ Combined patch and interpatch box plots of $\mathrm{EPSC}_{\mathrm{PV}} / \mathrm{EPSC}_{\mathrm{PN}}$ ratio in $\mathrm{dLGN} \rightarrow \mathrm{V} 1$ and $\mathrm{LP} \rightarrow \mathrm{V} 1$ pathways. (AG) Wilcoxon signed-rank test.

Figure 5. Tangential slices: sCRACM-mapping of $L M \rightarrow V 1$ input to $L 1$ onto L2/3 PNs and PVs in patches and interpatches. Same conventions as in Figure 3. (A) Venus labeled $\mathrm{LM} \rightarrow \mathrm{V} 1$ projections in L1 and Alexa 594 hydrazide-filled pairs of L2/3 PNs and PVs in patch and interpatch. (B-E) Recordings from PNs and PVs in patches and interpatches obtained in the same slice. (Bi, Biii) Each trace represents average of EPSC $_{\text {SCRACM }}$ to laser stimulation of ChR2- 
expressing $L M \rightarrow V 1$ terminals. (Bii, Biv). Heatmaps of responses from $P N$ and $P V$ shown in (Bi, Biii). (C) Dots represent relative strength of $L M$ input to a pair of $L 2 / 3 P N$ s and $P V$ in patch. $(A$, Di-iv, E) Recordings from PNs and PVs in interpatch. Same conventions as in (B, C). (F) Distribution of LM input strength across dendritic arbor. Grey bars (number of non-zero EPSC pxels), PNs and PVs in patches and interpatches. Red bars, EPSC/pixel density in PVs and PNs of patches and interpatches. $(G)$ Box plots of strength of $L M \rightarrow V 1$ EPSCs from PVs and PNs in patches and interpatches. (C, E, F, G) Wilcoxon signed-rank test ( ${ }^{* * *} p<0.001$, ns=not significant).

Figure 6. Spatial clustering of PV neurons in V1. (A) Diagram of coronal section through L1-3 showing M2+ patches (P), M2- interpatches (IP), and the preferred location of cell bodies, dendrites and boutons of PV-expressing basket $(\mathrm{BC})$ and chandelier $(\mathrm{ChC})$ cells. Arrows indicate positon of tangential sections (1-3) shown in (B-K). (B-D) Section 1 (A), immunolabeled M2+ patches (cyan) overlap (arrows) with PVtdT-expressing processes (magenta). (E-G) Section 2 (A), M2- interpatches (aligned to section in B) overlap (arrows) with PVtdT processes. (H-K) Section 3 (A), M2- interpatches (aligned to section in B) overlap (arrows) with PVtdT cell bodies, dendrites and boutons. (L) Normalized (to patches) fluorescence intensity at top of $L 1$ (section 1) showing stronger PVtdT expression in patches. (M) Normalized (to patches) fluorescence intensity in L2 (section 3) showing stronger PVtdT expression in interpatches. KS test, mean \pm SD (L, M). (N) Biocytin-filled L2/3 PV+ BCs (coronal plane) showing that dendrites (blue) branch in L1B-2. Axons (red) of cells in P (left panel) and IP (right panel) branch preferentially near the cell body with little spread into neighboring IP or $P$ territories, respectively. The left panel shows a $P$ cell with an asymmetrical axon arbor, which is mostly contained within $P$ territory and shows sparse projections to IP. The elongated shape of the axonal arbor is caused by sectioning the oval-shaped $\mathrm{P}$ parallel to the long axis. PVs are rare in $\mathrm{P}$, but branch density is similar to that of PVs in IPs.

Figure 7 Distinct feedforward inhibition in patches and interpatches. (A, B) Diagram of dual whole cell patch clamp recordings in coronal V1 slices of uIPSC in pairs of L2/3 presynaptic $\mathrm{PV}$ and postsynaptic PN aligned with ChR2.Venus-expressing $\mathrm{dLGN} \rightarrow \mathrm{V} 1$ patches (solid green) and interpatches (green outline) in L1. Recordings from PNs were made with high $\left[\mathrm{Cl}^{-}\right]$internal solution to shift equilibrium potential of IPSCs to $0 \mathrm{mV}$ and record IPSCs at $-70 \mathrm{mV}$ as inward currents. Recordings from PVs were made with K-gluconate internal solution. (C) IR-DIC image showing paired recordings from L2/3 PN (black *, regular spiking shown below), and PV (red *, tdT expression shown in inset, fast spiking response below). (D, E) Recordings from synaptically 
connected PVs and PNs in patch (D) and interpatch (E). Spike fired by current injection in presynaptic PV (red trace). Voltage clamp recording of uIPSCs from postsynaptic PNs (black). Note that uIPSCs are larger in interpatches (E) than patches (D). uIPSCs are blocked by bath application of Picrotoxin (blue). The insets in (D) and (E) show that uIPSCs follow presynaptic spikes with delays consistent with monosynaptic connections. (F) Box plots of uIPSC recorded from PNs in patches and interpatches. Bath application of Picrotoxin abolished uIPSCs $\left({ }^{* * *} p<\right.$ 0.001, two-sample t-test). uIPSCs in interpatches are larger $\left({ }^{* * *} p<0.001\right)$. (G) The total unitary inhibitory charge (IPSQ) is larger $\left({ }^{* * *} p<0.001\right.$, two-sample t-test) in interpatches than patches. $(\mathrm{H})$ The connection probability between PVs and PNs in patches and interpatches is similar.

Figure 8. Stronger inhibition in interpatches than patches. (A, B) Diagram of recordings in coronal slices of reciprocal uIPSCs and UEPSCs in synaptically connected pairs of L/2/3 PNs and PVs aligned with ChR2.Venus-expressing dLGN $\rightarrow$ V1 patches (solid green) and interpatches (green outline) in L1. Recordings from PNs were made with high [ $\left.\mathrm{Cl}^{-}\right]$internal solution. Recordings from PV were done with K-gluconate solution in the pipette. (C, D) Monosynaptic uEPSCs (red trace) recorded from $P V$ in response to a single spike (black trace) fired by L2/3 PNs in patch (C) or interpatch (D). Note that UEPSC in patches and interpatches have similar amplitudes and are blocked by DNQX (green C, D). In the reverse connection uIPSCs in interpatches (D) were larger than in patches (C), and were blocked by Picrotoxin (blue $C, D)$. The insets in (C) and (D) show that in both directions $\mathrm{PV} \rightarrow \mathrm{PN}$ and $\mathrm{PV} \leftarrow \mathrm{PN}$ postsynaptic responses followed spikes with lag times indicating monosynaptic connections. (E, F) UEPSCs and uIPSCs recorded in pairs of PNs and $P V$ in patches and interpatches. In comparing (E) and (F), note that in most pairs uIPSCs in interpatches are larger than in patches. (G) Average monosynaptic uEPSCs from reciprocally connected $\mathrm{PV} \leftrightarrow \mathrm{PN}$ pairs are similar (ns, one-way ANOVA), whereas uIPSCs in interpatches are larger $\left({ }^{* *} p<0.001\right.$, one-way ANOVA). (H) I/E ratio of reciprocally connected $\mathrm{PV} \leftrightarrow \mathrm{PN}$ pairs across patches and interpatches. Note that $\mathrm{I} / \mathrm{E}$ balance in interpatches is tilted toward inhibition $\left({ }^{* * *} p<0.001\right.$, two-sample t-test). (I) Average charge of uEPSCs and uIPSCs in patches and interpatches, showing that excitatory charge transfer at $\mathrm{PN} \rightarrow \mathrm{PV}$ contacts in patches and interpatches is similar, whereas the inhibitory charge transfer at $P V \rightarrow P N$ contacts in interpatches is larger ( ${ }^{* \star *} p<0.001$, one-way ANOVA, Bonferroni correction for multiple comparisons). (J) I/E ratio showing that in reciprocally connected $P V \leftrightarrow P N$ pairs in interpatches, uEPSCs are more strongly ( ${ }^{* *} p<0.01$, two-sample t-test) opposed by uIPSCs. $(K)$ The reciprocal connectivity within patches and interpatches is 2.5 -fold higher than between $P$ and IP. (L) The onset latency of uIPSC 
in patches and interpatches is similar. (M) The rise time of uIPSCs in interpatches is faster than in $P\left({ }^{* * *} p<0.001\right.$, two-sample t-test).

\section{SUPPLEMENTAL INFORMATION}

Figure S1. Intensity maps of $\mathbf{d L G N} \rightarrow \mathbf{V} \mathbf{1}$ and $L P \rightarrow \mathbf{V} \mathbf{1}$ projections. $(A, B)$ Distribution of fluorescence intensity of $M 2 t d T(A)$ and $d L G N \rightarrow V 1$ input $(B)$ (same sections as in Figure 1A, B). Patches are outlined by white lines (top 33\% quantile), interpatches by cyan lines (bottom 33\% quantile). (C, D) Interdigitating maps of $d L G N \rightarrow V 1(C)$ and $L P \rightarrow V 1$ (D) projections to $L 1$ (same section as in Figure 1I, K).

Figure S2. Cholinergic axons preferentially innervate M2 patches. (A, B) Tangential section through V1 showing double immunofluorescence of M2 patches (magenta) and ChAT axons (green) in L1. (C) Merge of (A) and (B). (D) Normalized (to patches) fluorescence intensity showing stronger ChAT expression in patches. KS test, mean \pm SD.

Figure S3. Development of M2 patches. (A, B) Tangential section through V1 showing patchy expression of $\mathrm{M} 2$ in L1 of 4 day-old Chrm2tdT mouse (A). At higher magnification membrane-bound M2 expression in L2/3 shows rings of crossectioned dendritic bundles (arrows) occupying the spaces between unstained cells bodies (B). (C) Patchy pattern of M2 immunostaining in L1 of $\mathrm{V} 1$ of $\mathrm{P} 10$ C57BL/6J mouse.

Figure S4. Recordings of L2/3 PVs and PNs in patches and interpatches. (A-C) Confocal z-stack $(200 \mu \mathrm{m})$ of tangential slice through L1-2 of V1 showing Alexa 594 hydrazide-filled pairs of PVs and PNs (red) aligned with Venus-expressing patch of LGN $\rightarrow$ V1 input to L1 (green) and Venus-negative interpatch. (B) Same slice as in (A) labeled with an antibody against M2. (C) Overlay of (A) and (B). (D) Alexa 594 hydrazide-filled interpatch-PVs and -PNs shown in (A) with apical dendrites branching preferentially in interpatches (unshaded regions). (E) Patch-PVs and -PNs shown in (A) with dendrites preferentially branching in patches (shaded regions). (F-J) Same format as $(A-E)$, except that the Venus-expressing patches represent $L M \rightarrow V 1$ inputs. Note that similar to dLGN projections, LM inputs overlap with M2 patches $(H)$. 


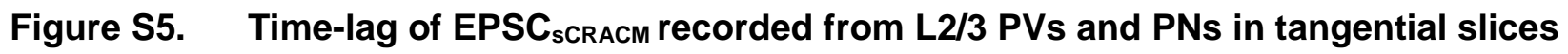
of $\mathbf{V 1}$. $(A, B)$ The time to peak after photo-stimulation of $d L G N \rightarrow V 1$ and $L M \rightarrow V 1$ inputs to $L 1$ showing faster ( ${ }^{*} p<0.05$, two-sample t-test) responses in PVs (red) than PNs (grey).

Figure S6. Clustering of PVtdT fibers in V1. Tangential section though L1 (70 $\mu \mathrm{m}$ below pial surface) of V1 of PVtdT mouse, showing clusters of tdT labeled dendrites and axons. Arrows points to branched blood vessel. Lateromedial area (LM), anterolateral area (AL), rostrolateral area $(R L)$, anteromedial area (AM), posteromedial area (PM), Mediomedial area (MM), restrosplenial agranular area (RSDagl), primary auditory area (AUDp), Somatosensory barrel cortex (SSp-bdf).

Figure S7. Preferential VGAT expression in interpatches. (A-D) Tangential sections through L1B-2 of V1 of VGAT-EYFP mouse. (A, B) Patchy dLGN $\rightarrow$ V1 inputs to L1 labeled by tracing with AAV2/1hSyn.tdTomato.WPRE.bGH (A) and corresponding contour map of patches (white lines) and interpatches (cyan lines) (B). (C-E) Patchy VGAT expression (C) and corresponding contour map (D) showing preferential expression in interpatches (cyan lines). (E) Merge of $A$ and $C$ shows interdigitating pattern. (F) Fluorescence intensity of VGAT-EYFP in interpatches relative to dLGN input to patches.

Figure S8. Ankyrin G immunolabeled axon initial segments (AIS) of L2/3 PNs aligned with M2 patches are densely innervated by PVs. (A) M2 immunolabeling (white) in parasagittal section through V1 of PVtdT (magenta) mouse. Patch (P). Interpatch (IP). (B) Same section as in (A) showing Ankyrin G immunolabeling of AIS (green) and M2 expression (white). Boxes indicate regions in L2/3 aligned with patches and interpatches in which PV innervation of AIS were counted. (C) Overlay of (A) and (B). (D) Confocal image showing putative contacts of PV boutons (magenta) with Ankyrin G labeled AIS (green). (E) Number of AIS in L2/3 underneath patches and interpatches. Length density of PV contacts onto L2/3 AIS in patches and interpatches. Paired t-test, ${ }^{* *} p<0.01$.

\section{Supplemental Experimental Procedures}

\section{Animals}

Experiments were performed on male and female, C57BL/6J, PV-Cre (Bg.129P2-Pvalb ${ }^{\text {tm(cre)Arbr/J) }}$

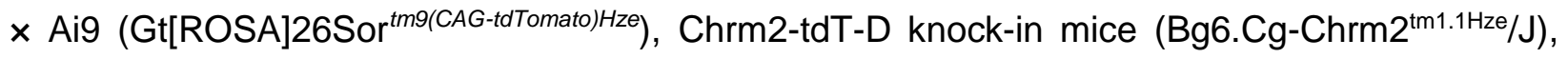
Chrm2 M2R-/- (B6N.129S4(Cg)-Chrm2 $\left.{ }^{\text {tmJwe }} / J\right)$ and VGAT-ChR2-EYFP (B6.Cg-Tg(Slc32a1- 
COP4*H134R/EYFP)8Gfng/J) mice. For anatomy we used 4-10 day old and $>46$ day old animals. Slice recordings were done in 34-46 day-old animals. Thalamocortical and intracortical connections to V1 were visualized by axonal tracing with AAV. sCRACM mapping of long-range input to PNs and PVs and recordings of UEPSCs and uIPSCs between synaptically connected pairs of PNs and PVs were performed in acute slices of V1. All experimental procedures were approved by the Institutional Animal Care and Use Committee at Washington University.

\section{Tracing of connections}

Connections were traced anterogradely by intracerebral injection of AAV2/9.CAG.ChR2.Venus.WPRE.SV40 (Vector Core, University of Pennsylvania), AAV2/1.hSyn.EGFP.WPRE.bGH (Vector Core, University of Pennsylvania) and/or AAV2/1hSyn.tdTomato.WPRE.bGH (Allen Institute) in 18-20 day-old or 8-12 week- old mice. Animals were anesthetized with Ketamine/xylazine (86 mg. $\left.\mathrm{kg}^{-1} / 13 \mathrm{mg} \cdot \mathrm{kg}^{-1}, \mathrm{IP}\right)$. Analgesia was performed with Buprenorphine $(0.1 \mathrm{mg} / \mathrm{kg}, \mathrm{SQ})$. The eyes were protected with ophthalmic ointment. All surgical procedures were performed in a stereotaxic apparatus. Injections (46 nl) were made with glass pipettes (tip diameter 15-25 $\mu \mathrm{m}$ ) connected to a Nanoject II injector. Stereotaxic injections were made into the (in $\mathrm{mm}$ ): dLGN (2.35 posterior of bregma, 2.15 lateral of midline and 2.55 below the pial surface), LP (1.85 posterior of bregma, 1.25 lateral of midline and 2.65 below the pial surface), higher visual cortical lateromedial area, LM, (1.4 anterior to transverse sinus, 4.1 lateral to midline, 0.3- 0.5 below the pial surface) and posteromedial area, PM, (1.9 anterior to transverse sinus, 1.6 lateral to midline, 0.3-0.5 below the pial surface). Postsurgical survival was 2-3 weeks.

\section{Immunostaining}

Mice were overdosed with Ketamine/xylazine (170mg. $\left.\mathrm{kg}^{-1}\right)$ and transcardially perfused with heparinized phosphate-buffered saline (PBS), followed by $1 \%$ paraformaldehyde (PFA). Brains were extracted from the skull, the left cortical hemisphere was removed, flat mounted, postfixed overnight in 4\% PFA and cryoprotected in 30\% sucrose. Tangential or parasagittal sections were cut at $40 \mu \mathrm{m}$ with a freezing microtome. Sections were washed in $0.1 \mathrm{M}$ phosphate buffer (PB), treated in blocking solution containing 10\% normal goat serum (NGS), and $0.1 \%$ Triton X-100 in PB. Immunolabeling was performed by incubating sections for 48 hours at $4^{\circ} \mathrm{C}$ with primary antibodies against M2 muscarinic acetylcholine receptor (1:500 rat monoclonal, MAB367 Millipore) or anti-Ankyrin G (1:1000, mouse monoclonal, clone N106/36, NeuroMab). After washing, sections were treated with Alexa Fluor 647-labeled goat anti-rat IgG secondary antibody 
(1:500 in 10\% NGS; A21247 Invitrogen) or donkey-anti-mouse-Cy5 (1:500, 715-175-151, Jackson Immuno Research). Cholinergic fibers were identified with an antibody against choline acetyl transferase (1:1000, goat anti-ChAT, Millipore AB144P), detected with a biotinylated donkey-anti goat secondary antibody (1:200, Millipore AP180B) and visualized with NeutrAvidin Oregon Green 488 (1:400, ThermoFisher A6374). Sections were mounted onto glass slides, coverslipped in PB or Aqua Poly/Mount (Polysciences) and imaged under a fluorescence microscope (Nikon 80i) equipped with a CCD camera (CoolSnap EZ, Roper Scientific or Infinity3S-URM, Lumenera). Confocal imaging was performed with an Olympus Fluoview (FV1200) microscope. The specificity of the M2 primary antibody was validated in C57BL/6J-M2I- mice (Gomeza et al., 1999) in which we saw no detectable staining.

\section{Slice preparation}

Slices of V1 were prepared from 34-46 days-old virus-injected mice. The slices were either cut in the tangential or the coronal plane. Tangential slices were optimally suited for identifying repeating clusters of ChR2.Venus-labeled thalamocortical and intracortical inputs to L1 and preserving the complete dendritic arbors of PNs and PVs. Mice were decapitated under isoflurane $(2 \%$ in oxygen) anesthesia. The brain was rapidly removed from the skull and submerged in ice-cold cutting solution aerated with $95 \% \mathrm{O}_{2} / 5 \% \mathrm{CO}_{2}$ containing (in $\mathrm{mM}$ ): 240 sucrose; $2.5 \mathrm{KCl}, 1.25$ $\mathrm{NaH}_{2} \mathrm{PO} 4,2.1 \mathrm{NaHCO}_{3}, 7 \mathrm{MgCl}_{2}, 0.5 \mathrm{CaCl}_{2}, 10$ glucose, adjusted with $\mathrm{NaOH}$ to pH 7.35. Next, the cerebellum and anterior third of the brain were removed. With the cut rostral surface towards the base, the lower part of the brain was resected parallel to the surface of V1 and the tissue block was mounted with the cut-side down onto the specimen plate. Single tangential slices (350 $\mu \mathrm{m})$ were cut in ice-cold cutting solution on a Vibratome (Leica VT 1200). Coronal slices were prepared as described previously (Yang et al., 2013). Slices were kept in a holding chamber in which they were submerged in oxygenated artificial cerebrospinal fluid (ACSF; containing [in $\mathrm{mM}$ ]:125 NaCl, $2.5 \mathrm{KCl}, 1.25 \mathrm{NaH}_{2} \mathrm{PO}_{4}, 25 \mathrm{NaHCO}_{3}, 1 \mathrm{MgCl}_{2}, 2 \mathrm{CaCl}_{2}, 25$ glucose, pH 7.35) for 1 hour at $32^{\circ} \mathrm{C}$ before transferring them to the recording chamber maintained at room temperature $\left(22-24^{\circ} \mathrm{C}\right)$.

Clustered projections were also readily identified in coronal slices which are optimal for preserving connections across layers. The procedures of preparing coronal slices were identical to those for tangential slices, except that the injected hemisphere was mounted with the coronally cut frontal surface down and serial slicing was from the posterior pole.

\section{Subcellular Channelrhodopsin-2 assisted circuit mapping (sCRACM)}


For recording, slices were transferred to a submersion chamber mounted on the stage of a modified upright fluorescence microscope (Nikon Eclipse FN1) equipped with a CCD camera (Retiga-2000C; Qimaging). Slices where perfused (1.5 $\mathrm{ml} / \mathrm{min}$ ) with recirculating oxygenated ACSF $\left(22-24^{\circ} \mathrm{C}\right)$. Whole cell patch clamp recordings were obtained from pairs of tdT-expressing PVs (identified with fluorescence optics) and nearby (within $<40 \mu \mathrm{m}$ ) unlabeled PNs cells (identified with DIC-IR optics) in L2/3 of V1. Cell pairs were either in-register (i.e. within patches) or out-of-register (i.e. within interpatches) with ChR2.Venus-labeled patches of axons projecting from the dLGN, LP or area LM and terminating in L1 of V1. For recording in tangential slices, neurons were approached from the cut surface of the slice, which was mounted with the pial surface facing down. In tangential and coronal slices, recordings were made 30-120 $\mu \mathrm{m}$ below the surface of the slice. Electrical signals were sampled at $10 \mathrm{kHz}$ by Multiclamp 700B amplifiers (Molecular Devices), digitized (NI USB 6363; National instruments) and acquired using Matlabbased (MathWorks, Natick, MA) Ephus software (Suter et al., 2010). Electrodes were pulled from borosilicate glass capillaries (G150F-4, Warner Instruments). Pipettes were filled with (in $\mathrm{mM}$ ): 128 potassium gluconate, $4 \mathrm{MgCl}_{2}$, 10 HEPES, 1 EGTA, $4 \mathrm{Na}_{2} \mathrm{ATP}, 0.4 \mathrm{Na}_{2} \mathrm{GTP}$, 10 sodium phosphocreatine, 3 sodium L-ascorbate, 0.02\% Alexa 594 hydrazide (Invitrogen), pH 7.25, 290 mOsm. The pipette resistance was 3-5 M . The liquid junction potential was not corrected. The seal resistance was $>2 \mathrm{G} \Omega$. Recordings with access resistance of $>20 \mathrm{M} \Omega$ were excluded from the study. Neuron type was assessed by recording spiking patterns (i.e. fast for PVs, regular for PNs) in response to 300 ms pulses of hyperpolarizing and depolarizing current in current clamp mode. For sCRACM mapping (Petreanu et al., 2009), EPSCs were recorded in voltage clamp at a holding potential of $-70 \mathrm{mV}$, with tetrodotoxin $(1 \mu \mathrm{M})$ and 4-aminopyridine (4-AP; $100 \mu \mathrm{M}$; Tocris Bioscience) in the bath to block action potentials and fast repolarizing potassium currents, respectively. After recording, slices were fixed in 4\% PFA, cleared in 10\% sorbitol, mounted on glass slides in Aqua Poly/Mount, imaged under a confocal microscope (Olympus, Fluoview FV1200) and reconstructed in 3D, using ImageJ.

\section{Photostimulation}

Photostimulation was performed with a blue laser (473 nm; CrystaLaser). The light was reflected by a fixed set of mirrors onto galvanometer scanners (Cambridge Scanning) that controlled beam position. The light then passed through an air objective (4 PlanApo, NA 0.2; Nikon), which at 0.25 $\mathrm{mW} / \mathrm{cm}^{2}$ laser power formed a beam at half maximal intensity with a diameter of $\sim 20 \mu \mathrm{m}$ in the specimen plane. The durations and intensities of the light pulses were controlled with a Pockels cell (ConOptics) and a shutter (LS6, Uniblitz). Because the proportion and labeling intensity of 
ChR2-expressing axons varied across slices and animals, the laser power $\left(0.25-1 \mathrm{~mW} / \mathrm{cm}^{2}\right)$ was adjusted in every slice to evoke EPSC. The laser power was constant for all recordings made that day. Recordings in V1 were performed from pairs of nearby PNs and PVs in patch and interpatch regions of L2/3. Each trial consisted of $100 \mathrm{~ms}$ baseline, followed by the photostimulus (1-2 ms) and $300 \mathrm{~ms}$ of response. Photostimulation was performed in an $8 \times 8 \mathrm{grid}$ in which individual points were spaced $75 \mu \mathrm{m}$ apart and the grid was aligned with the recorded soma at the center. For mapping in coronal slices, one side of the grid was aligned with the pial surface. The stimulation sequence was pseudorandom allowing maximal intervals between nearby stimulation sites. sCRACM maps were generated from 3-5 repetitions per neuron.

\section{Recordings from synaptically connected pairs}

To examine the presence and strength of synaptic connections between PVs and PNs in patches and interpatches we recorded from synaptically connected pairs in superficial L2/3 of V1. Patchy projections to L1 were identified as clusters of Venus-expressing axon terminals, labeled by tracing dLGN $\rightarrow \mathrm{V} 1$ inputs with AAV2/9.CAG.ChR2.Venus. Recordings were obtained from cell pairs (< $40 \mu \mathrm{m}$ apart) aligned with patches or interpatches in coronal slices. Responses in PVs were recorded with pipettes (4-6 $\mathrm{M} \Omega$ resistance) filled with (in $\mathrm{mM}$ ): 128 potassium gluconate, 4 $\mathrm{MgCl}_{2}$, 10 HEPES, 1 EGTA, $4 \mathrm{Na}_{2}$ ATP, $0.4 \mathrm{Na}_{2} \mathrm{GTP}$, 10 sodium phosphocreatine, 3 sodium Lascorbate, $\mathrm{pH}$ 7.25, $290 \mathrm{mOsm}$. For recording PNs, pipettes were filled with a high $\left[\mathrm{Cl}^{-}\right]$solution containing (in mM): $145 \mathrm{KCl}, 10$ HEPES, $5 \mathrm{NaATP}, 0.2 \mathrm{NaGTP}$ and $5 \mathrm{EGTA}$, pH 7.3, adjusted with $\mathrm{KOH}, 285 \mathrm{mOsm}$. Under these conditions the reversal potential of IPSCs is $0 \mathrm{mV}$ and at -70 $\mathrm{mV}$ holding potential the currents flow inward (Luo et al., 2013). Monosynaptic uIPSCs were recorded by holding PNs at $-70 \mathrm{mV}$. ulPSCs were elicited by triggering single action potentials from presynaptic PVs with $2 \mathrm{~ms}$ depolarizing current pulses. The same stimulation/recording paradigm was used for eliciting and measuring UEPSCs, except that spikes were elicited from PNs and responses were recorded from PVs. Responses were averaged across 50-150 repetitions at $0.5 \mathrm{~Hz}$. The error due to the liquid junction potential was not corrected. Access resistance was monitored throughout the experiment. Cells whose series resistance was $>20 \mathrm{M} \Omega$ or varied $>25 \%$ for the duration of the experiment were excluded from the analysis. Series resistance errors were not compensated. To block spontaneous polysynaptic NMDA receptormediated excitatory currents, CPP ((RS)-3-(2-carboxypiperazin-4-yl)-propyl-1-phosphonic acid, $50 \mu \mathrm{M}$, Tocris) was applied in the bath. uIPSCs were blocked by bath application of the $\mathrm{GABA}_{\mathrm{A}^{-}}$ receptor antagonist Picrotoxin $(50 \mu \mathrm{M}$, Tocris). UEPSCs were blocked by bath application of the AMPA-receptors antagonist DNQX (6,7-dinitroquinoxaline-2,3-dione, $20 \mu \mathrm{M}$, Tocris). After 
recording, the slices were fixed in 4\% PFA, mounted on glass slides, cleared in $10 \%$ sorbitol and Alexa 594 hydrazide-filled neurons were imaged under the confocal microscope.

\section{Data analysis}

\section{Contour plots of patches and interpatches}

Automatic patch/interpatch definition followed the general procedures of Sincich and Horton (2005). Fluorescent images of spatially clustered M2 expression or virally traced projection patterns in L1 of V1 were high-pass filtered using an $80 \mu \mathrm{m}$ filter radius. Images were then blurred using a circular averaging filter of $30 \mu \mathrm{m}$ radius, with the 'fspecial' function in Matlab. All pixels in the resulting images were then divided into six intensity quantiles. The top two quantiles were considered to be patches and the bottom two interpatches. For statistical testing, images in matching fields of view were analyzed. Images were downsampled to have a pixel area of 150 $\mu \mathrm{m}^{2}$ each. A permutation test was then performed by shuffling fluorescent image pixels within the image and determining the ratio of resulting average patch intensity to average interpatch intensity, maintaining the original patch/interpatch borders derived from M2 or viral tracings. Patch/interpatch ratios in the original image outside the $95 \%$ bounds of the randomized distribution from 100,000 shuffling iterations were considered significant deviations from a 1:1 patch/interpatch intensity ratio.

\section{Quantification of fluorescence intensity}

The intensity of immunofluorescence of $\mathrm{M} 2$, fluorescently labeled projections tagged by viral axonal tracing, and fluorescence of transcribed tdT and EGFP genes were quantified in images of tangential sections through L1, acquired with a CCD camera (Lumenera Infinity3S-URM) and Metamorph NX2.0 software (Molecular Devices). Gray scale images were opened with Image J, background subtracted to correct for global non-uniformities in brightness and overlaid with contour maps of fluorescence intensity determined by a custom Matlab script. Pixel values in patches and interpatches were measured at multiple sites, normalized to the mean brightness of patches, binned and plotted as counts of normalized fluorescence intensity. Statistical comparisons of intensity distributions were made using the KS test.

\section{EPSCs and IPSCs}

The amplitude of significant responses was $>4$ times the SD of the baseline. Individual pixel values of sCRACM maps were computed from the mean EPSC amplitude in a 75 ms response 
window after the photostimulus. For each neuron, maps were averaged across 3-5 repetitions. These averages represent synaptic charge transfer. Because the responses were dominated by the current amplitude and small long-lasting currents were negligible, we have adopted the simplification introduced by Petreanu et al., (2009) and represent responses in pA instead of Coulomb. The charge value for each pixel in a $75 \mathrm{~ms}$ window was calculated using custom Matlab software. EPSC amplitudes were measured with reference to the soma at the center. To display the scaled magnitudes and spatial distributions of thalamocortical and intracortical inputs from LM to PNs and PVs, maps for each cell class were peak normalized within individual slices and displayed as heatmaps. Comparisons of inputs to PNs and PVs were made by plotting the average responses from pairs of PNs and PVs within layer 2/3 of the same slice and in the same patch or interpatch module. Thalamocortical and intracortical inputs to PNs and PVs recorded in the same layer and same slice were plotted against each other and the relative strengths of excitation was assessed by plotting the mean slope from zero. uIPSC lag time was calculated as the time delay from the onset of the presynaptic PV spike to the onset of the uIPSC recorded from the postsynaptic PNs. UIPSC rise time was measured as the delay between response onset and the peak.

\section{Confocal imaging and Neuron reconstruction}

Alexa Fluor 594 hydrazide-filled neurons were reconstructed posthoc and their location in Venusexpressing patches of dLGN or LM inputs determined by imaging under a confocal microscope (Olympus, Fluoview FV 1200), using a 30x silicone oil (UPLSAPO, 1.05 NA, Olympus) objective. Twelve bit $1024 \times 1024$ pixel images were taken at 1.5x digital zoom. Z-stacks were acquired at $0.80 \mu \mathrm{M} /$ section (Nyquist volume: $1.6 \mu \mathrm{M}$ ) across the thickness of the slice. Multicolor scanning was done in sequential and frame-by-frame mode. The images were acquired in separate high sensitivity detector channels for each fluorophore. The signals were acquired and averaged by Kalman's method to increase signal/noise ratio. The neurons were then traced and reconstructed by using the 'Simple Neurite Tracer' Plugin of Fiji (ImageJ). PNs were identified by the presence of dendritic spines, whereas PVs have aspinous, beaded dendrites.

Recorded neurons were filled with biocytin $(3 \mathrm{mg} / \mathrm{ml}$ ) which after fixing slices in $4 \%$ PFA was visualized by an $A B C$ reaction and intensification of the reaction product with $\mathrm{AgNO}_{3}$ and $\mathrm{HAuCl}_{2}$ (Yang et al., 2013). Filled neurons were reconstructed under a 40x oil objective using Neurolucida (MicroBrightField). 
The innervation density of PN-AISs by PVtdT expressing boutons was determined by confocal imaging with a $100 x$ oil immersion objective of z-stacks $(0.2 \mu \mathrm{m}$ step size $)$ in ROls $(65 \times 135 \mu \mathrm{m})$ aligned with $\mathrm{M} 2+$ patches and M2- interpatches. To minimize contamination by Ankyrin Gexpressing AIS of interneurons we focused the analysis on tapered, vertically $\left( \pm 3^{\circ}\right.$ relative to the pial surface) descending profiles. Appositions between boutons and AIS were scored as contacts if there was no detectable gap between pre-and postsynaptic elements and their association remained stable under image rotation.

\section{Statistics}

Statistical analyses were performed using Origin 9.1 (Origin Laboratory) or customized Matlab software. Normality was assessed using the Shapiro-Wilk normality test to select between parametric and nonparametric tests. Comparisons between two groups were performed with twotailed Student's $t$-test. Neighboring neurons that were recorded sequentially were considered pairs and subjected to a paired $t$-test. For comparisons across more than two groups, data were analyzed using one-way ANOVA followed by Bonferroni's post hoc analysis to correct for multiple comparisons. For data with non-normal distribution, nonparametric KS test was used. Significance was $p<0.05$. Data are mean \pm SEM, except when otherwise indicated as mean \pm SD. Box plots mean \pm SD. 
Figure 1

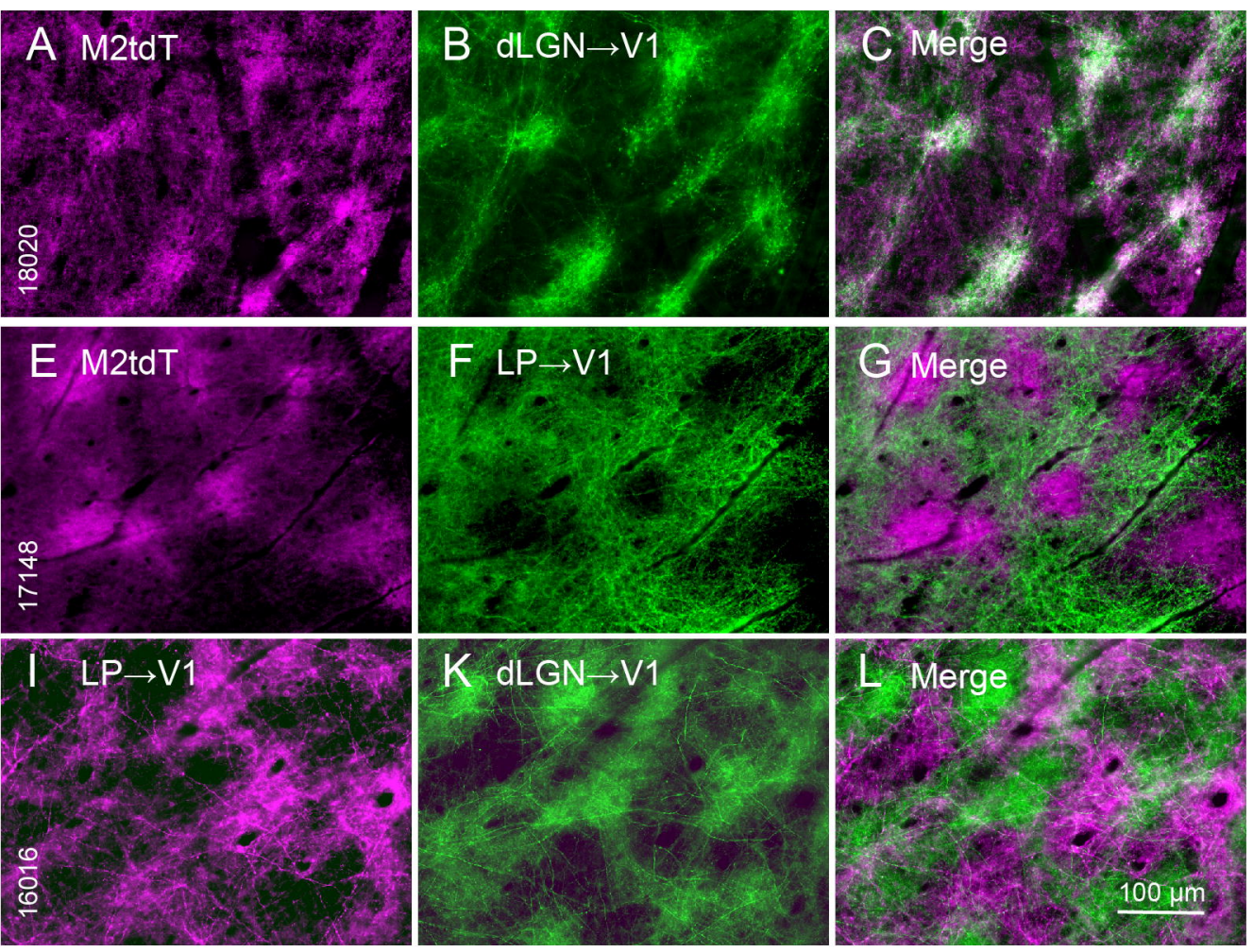

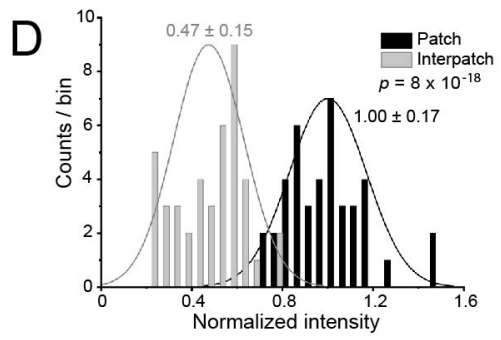

$\mathrm{H}$
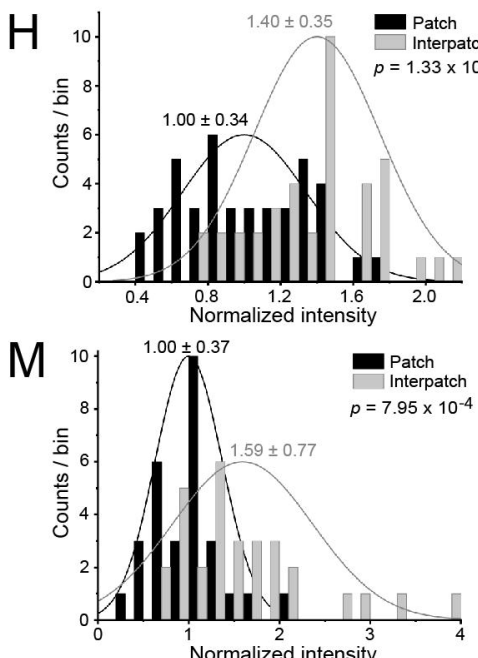


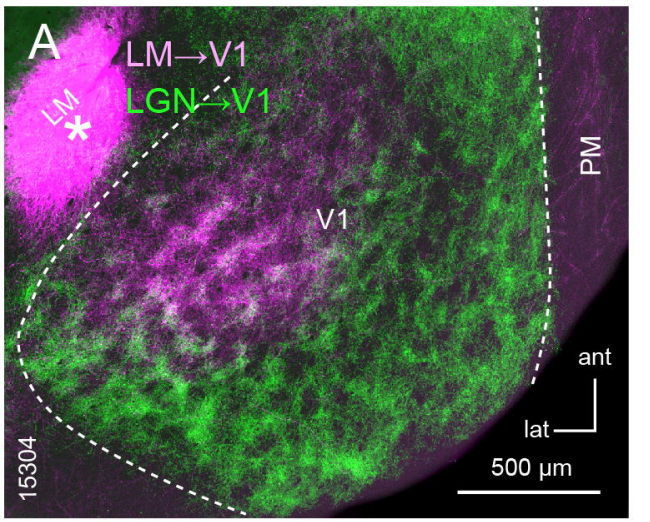

B $\quad \mathrm{LM} \rightarrow \mathrm{V} 1$

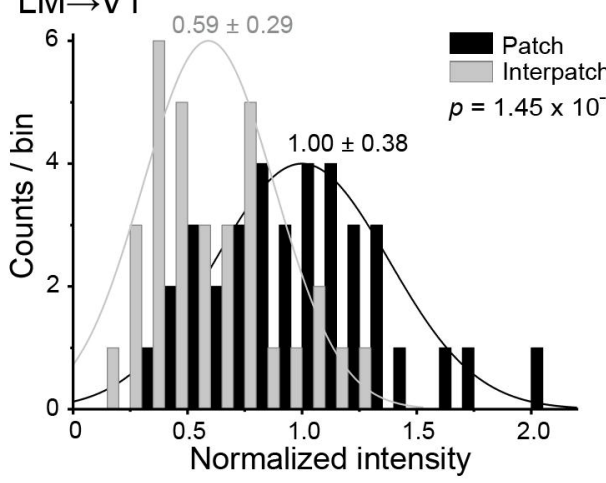

C M2tdT
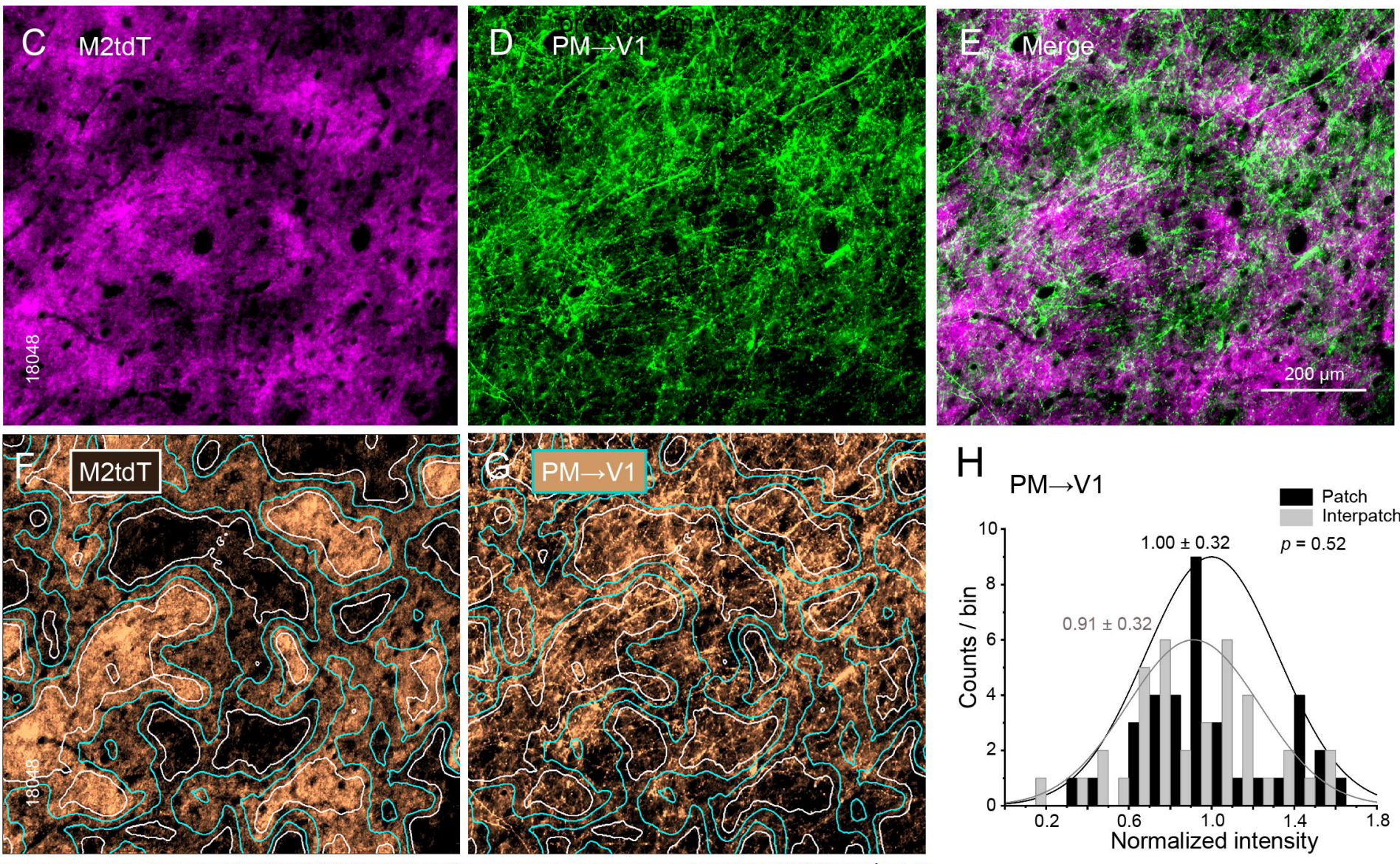

$\mathrm{PM} \rightarrow \mathrm{V1}$
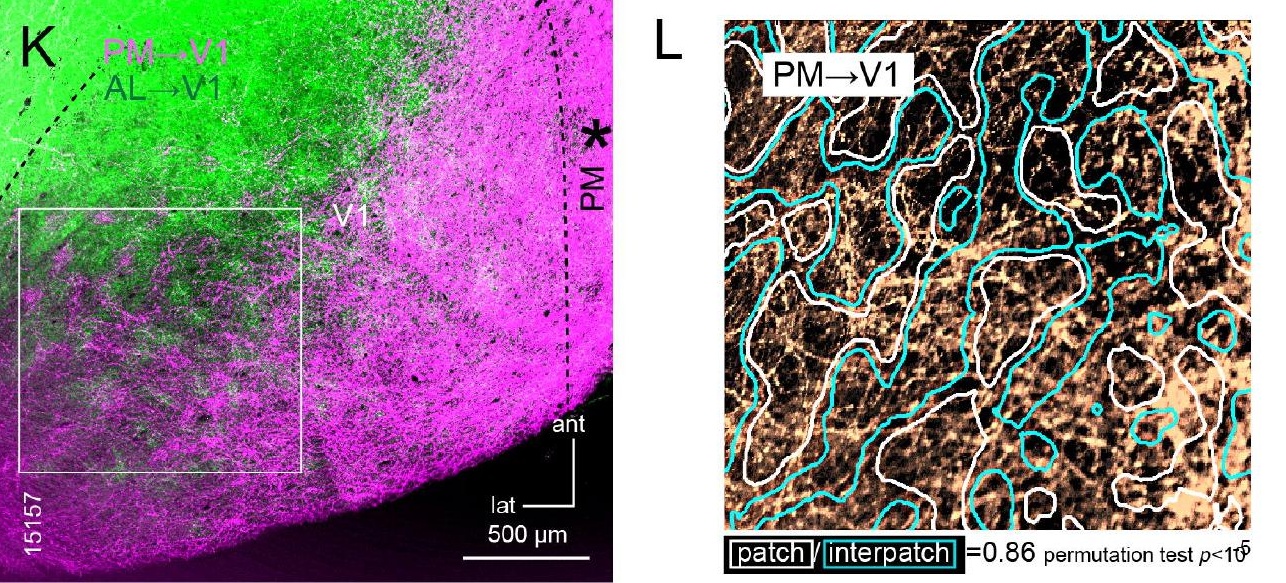


\section{Figure 3}

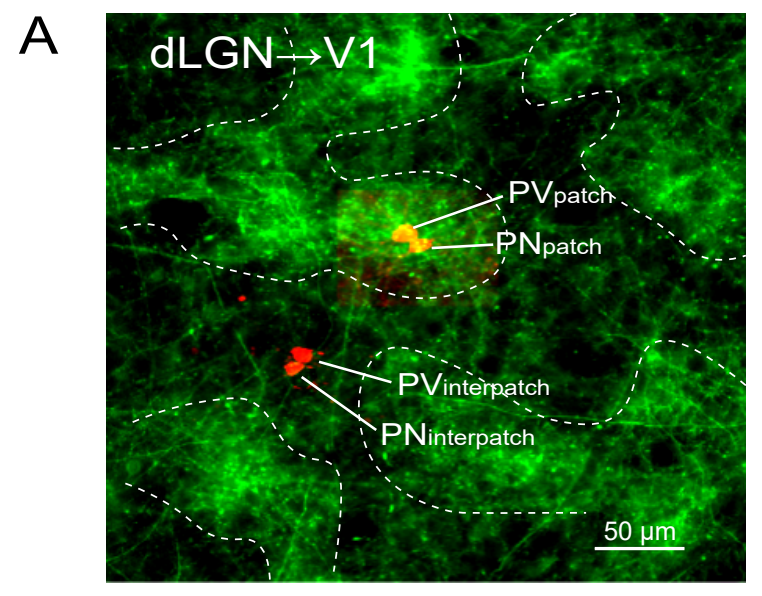

$\mathrm{B}$

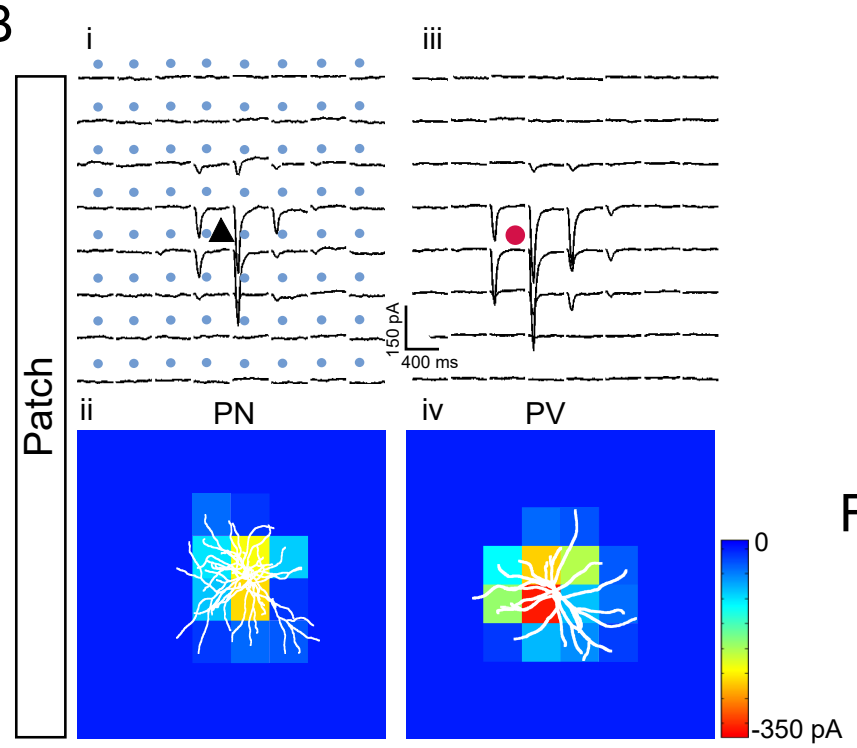

D

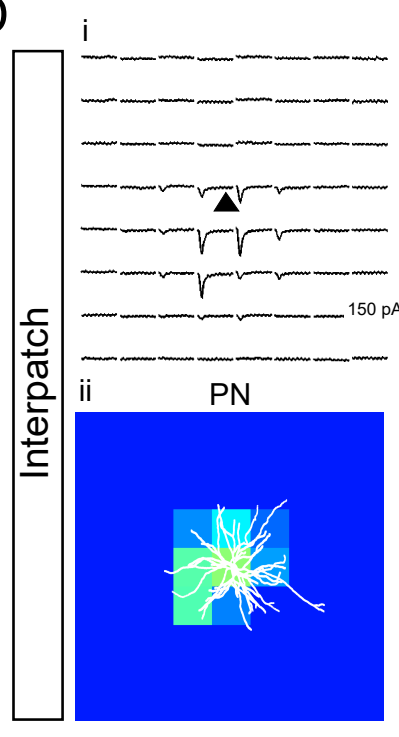

iii

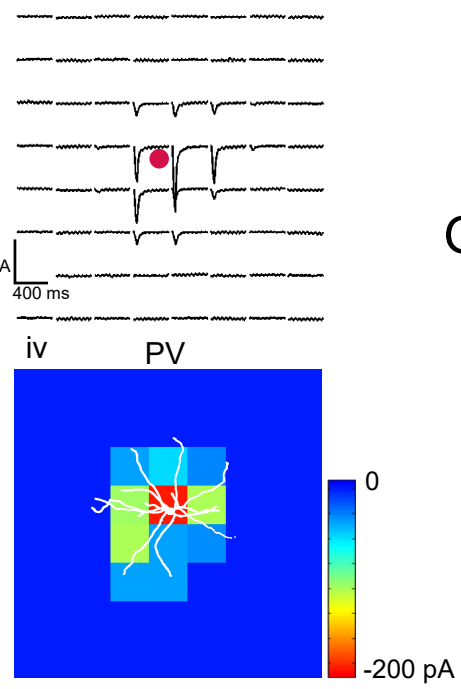

C

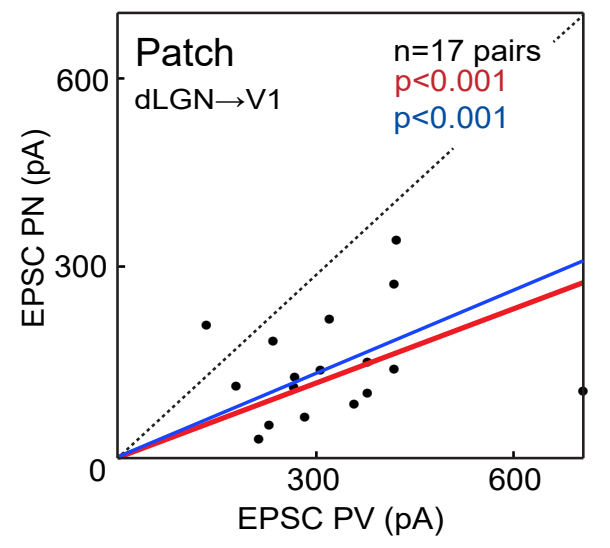

$E$

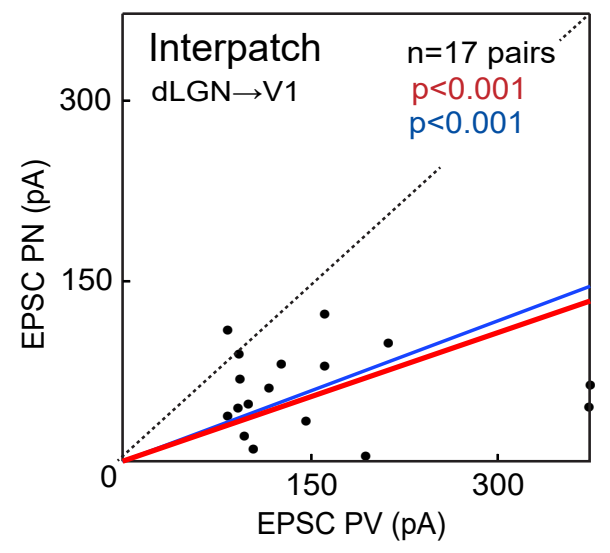

F

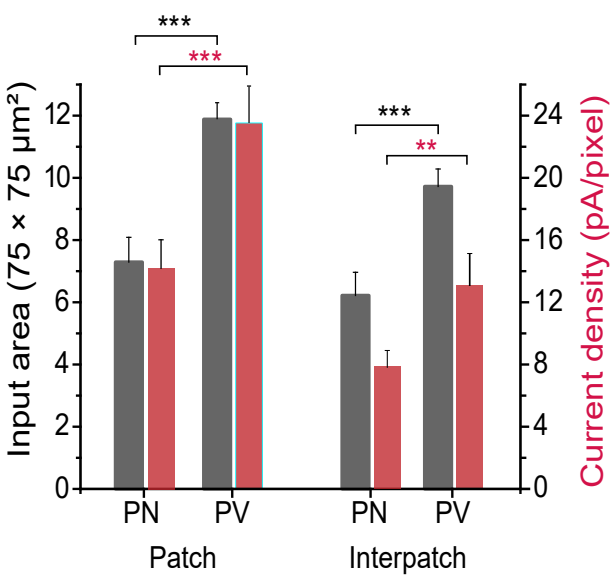

$G$

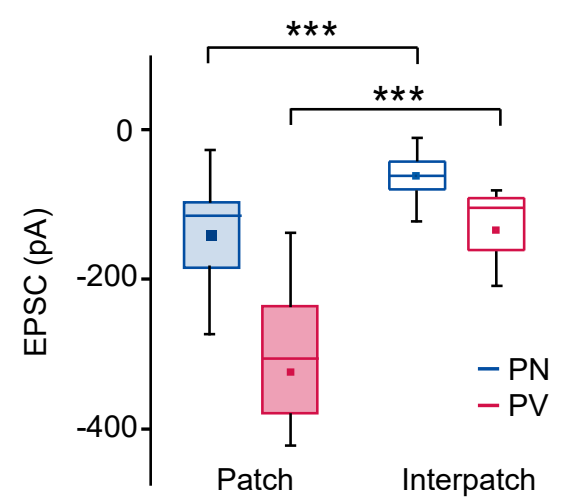




\section{Figure 4}
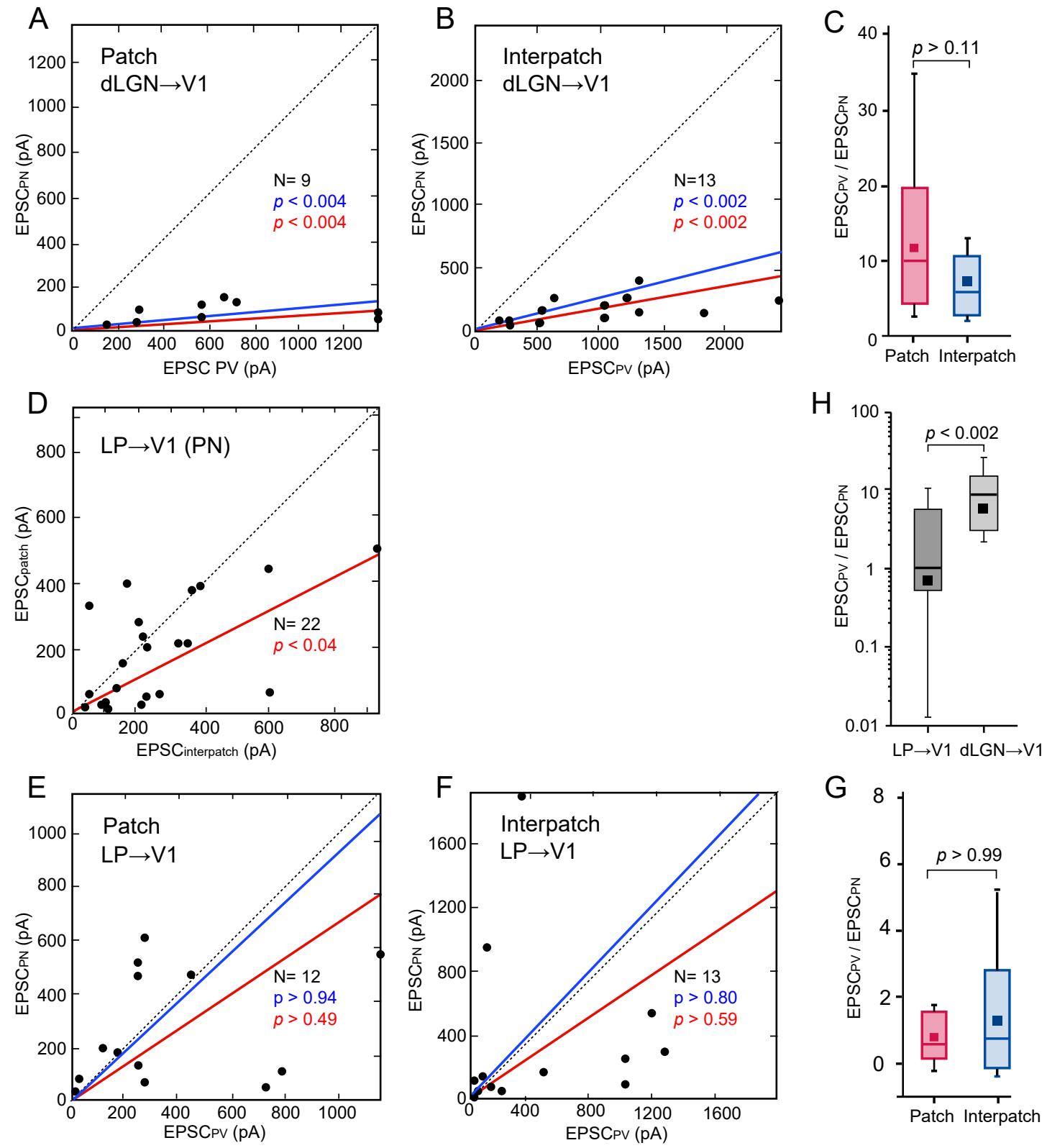
Figure 5

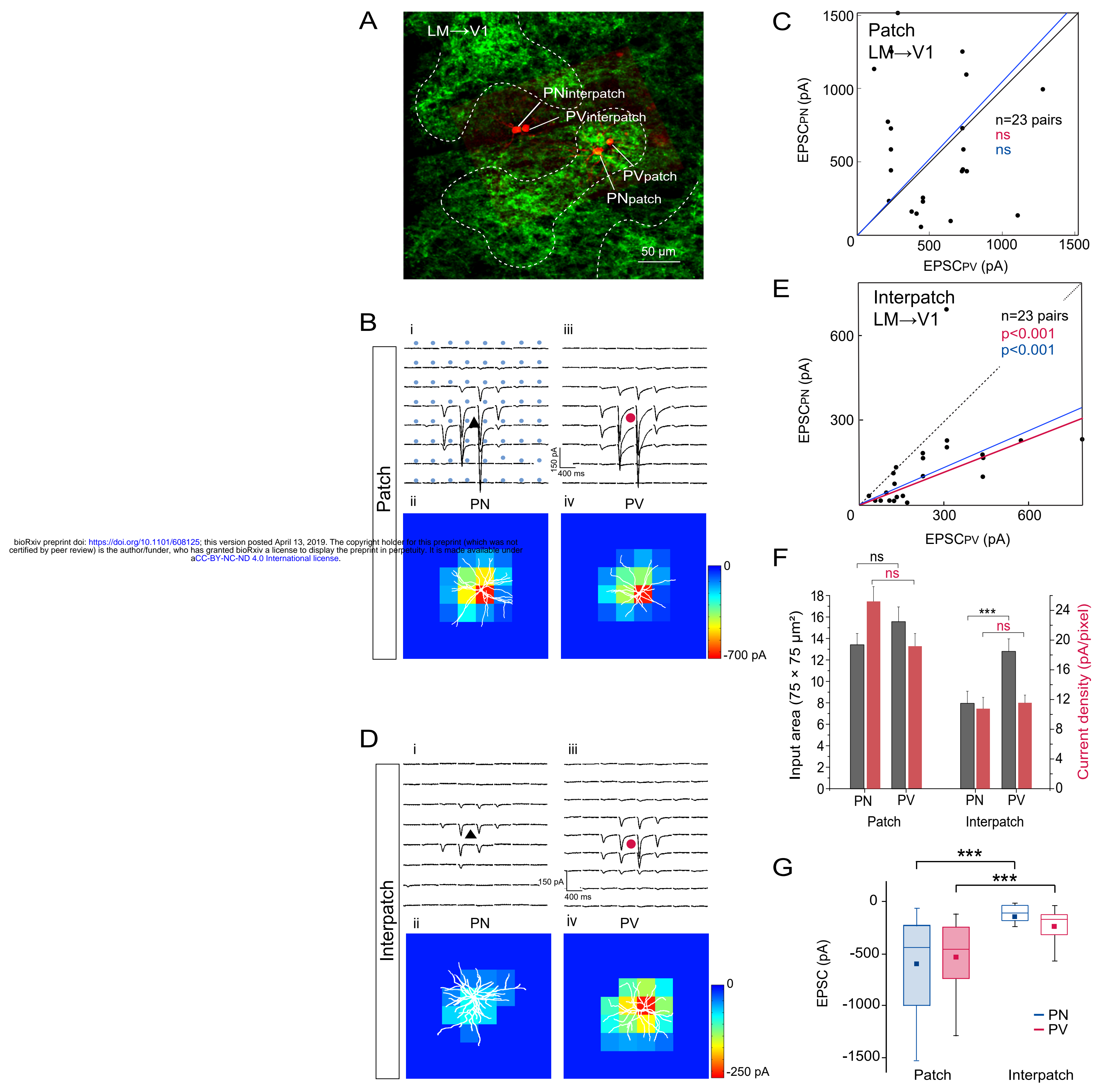


Figure 6
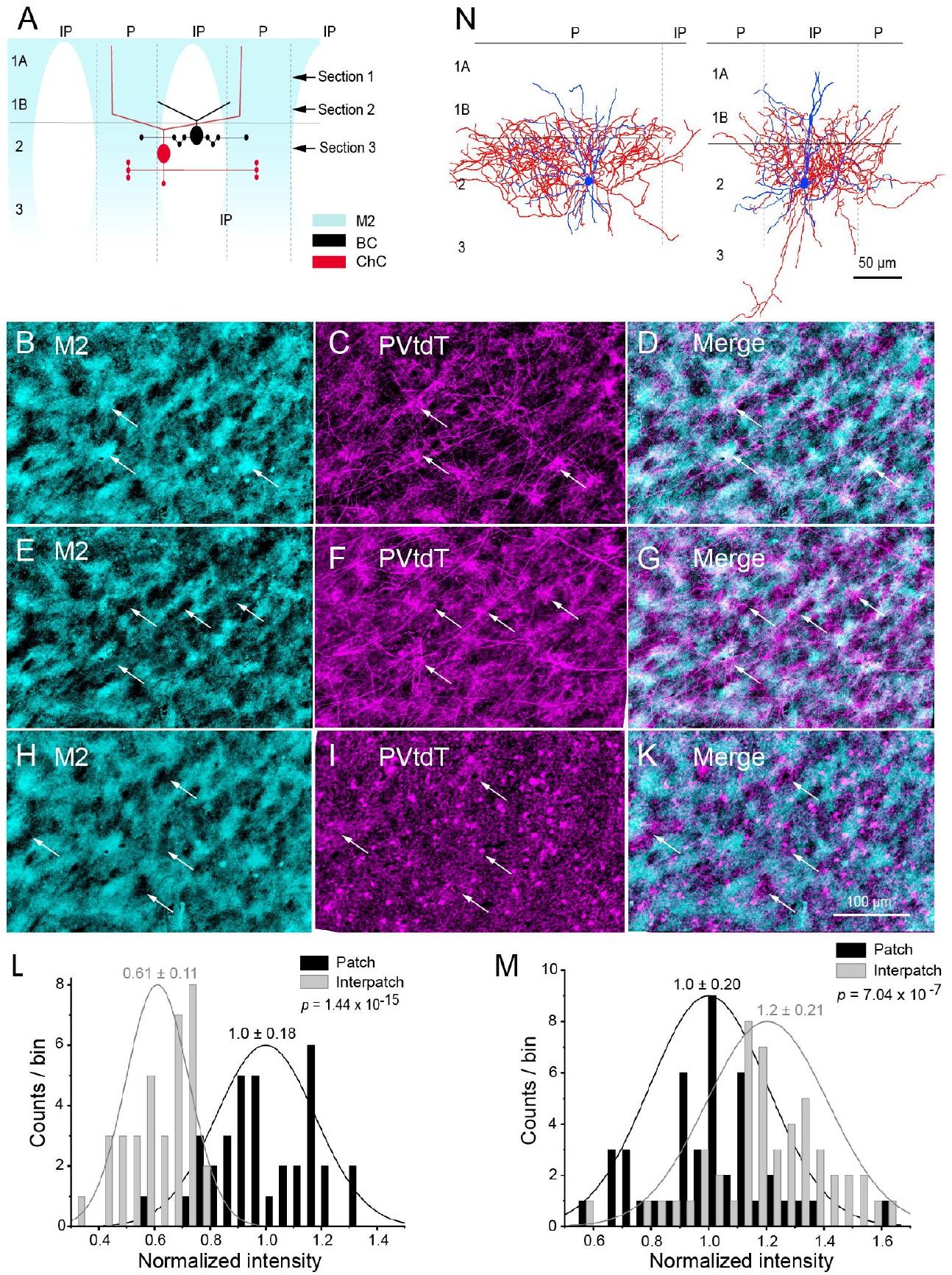


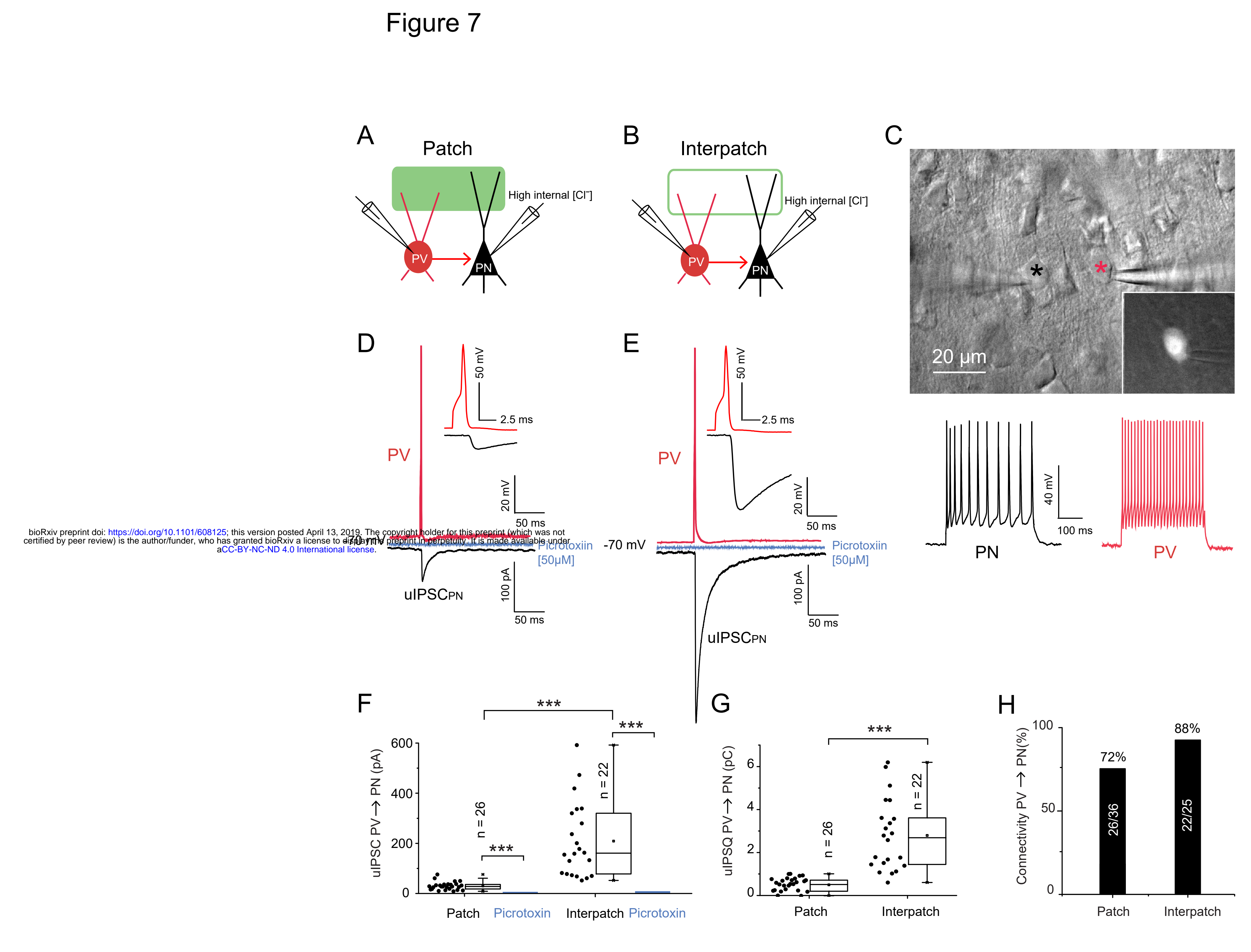


Figure 8

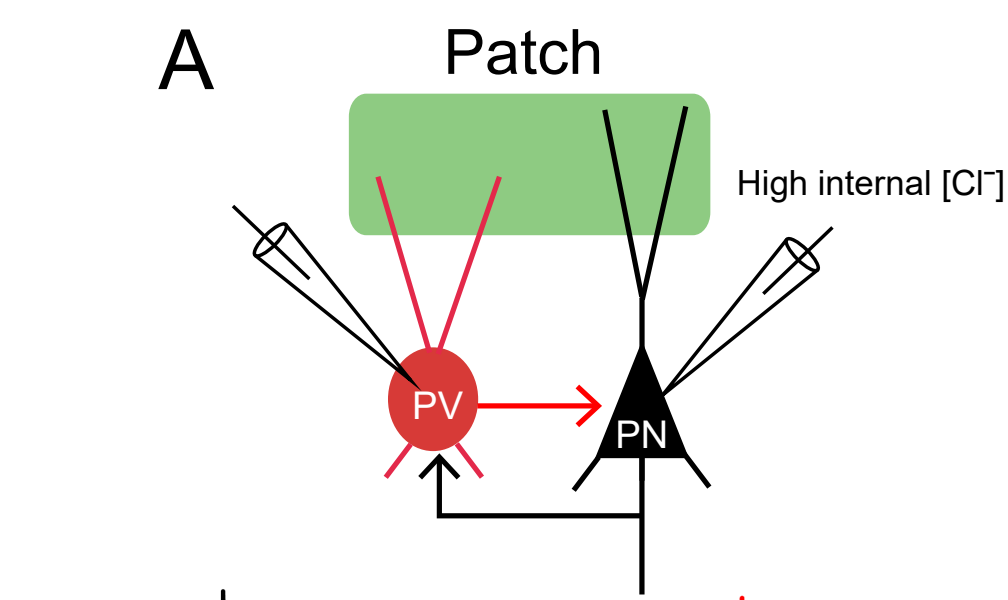

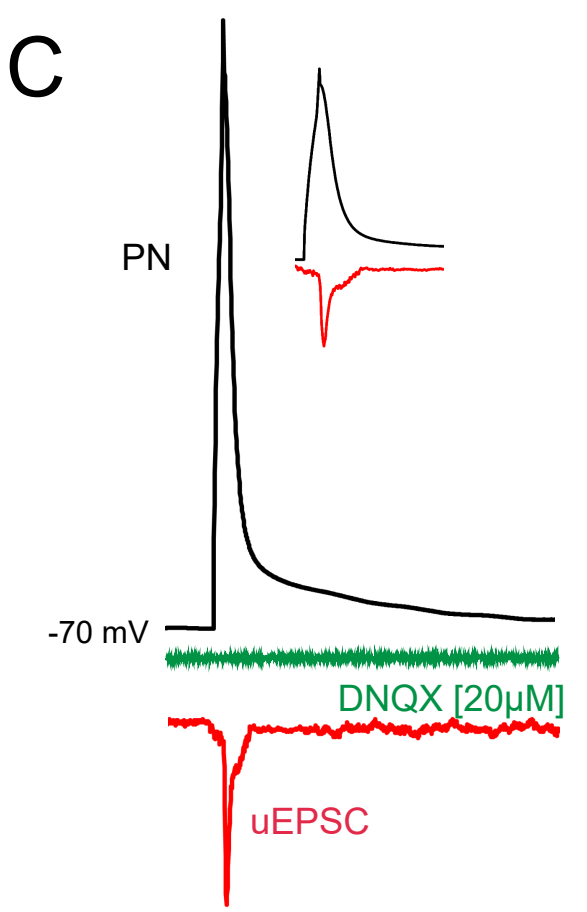

E

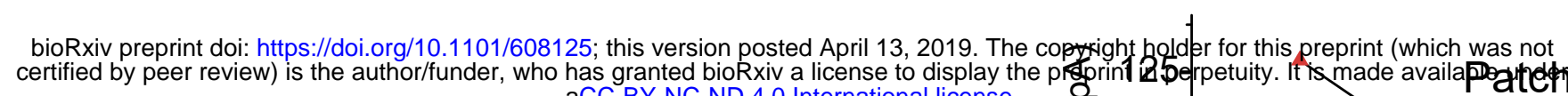

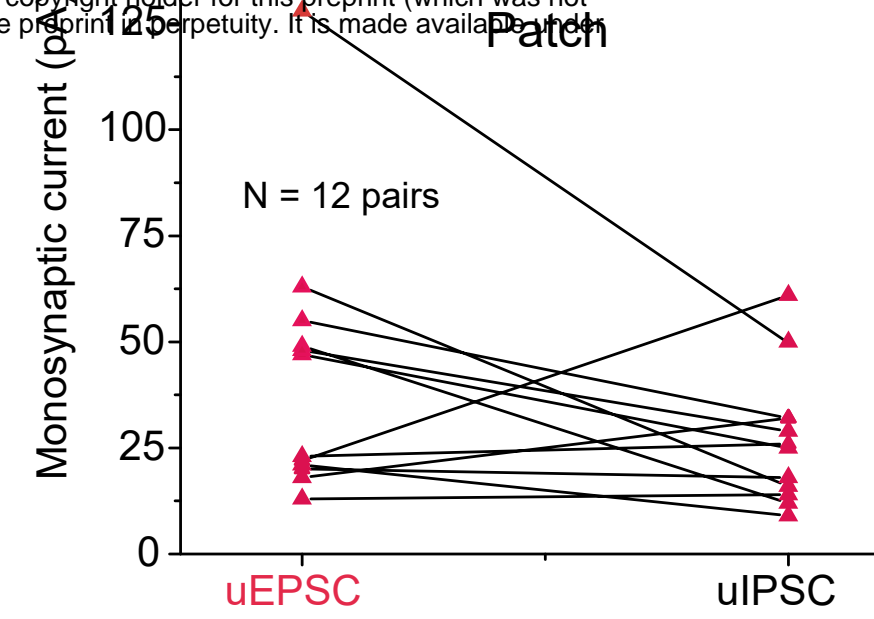

G
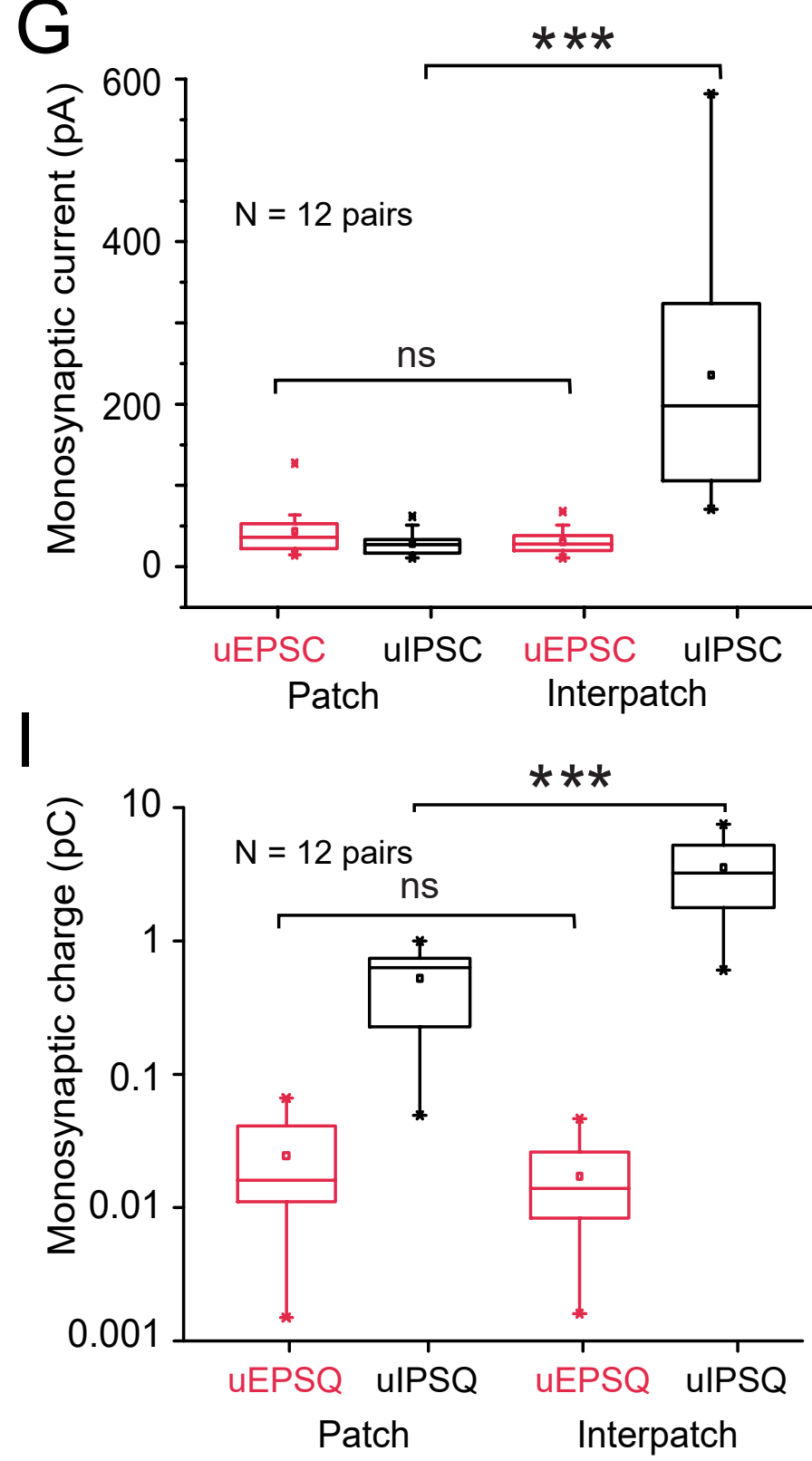

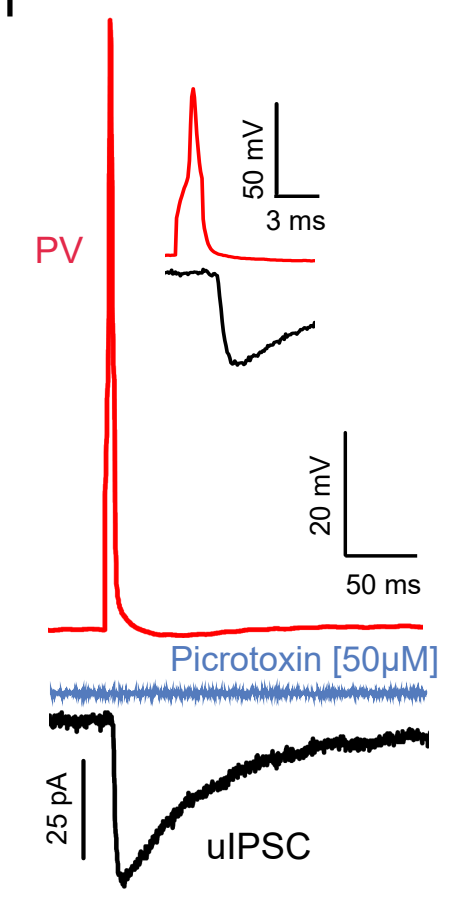

F

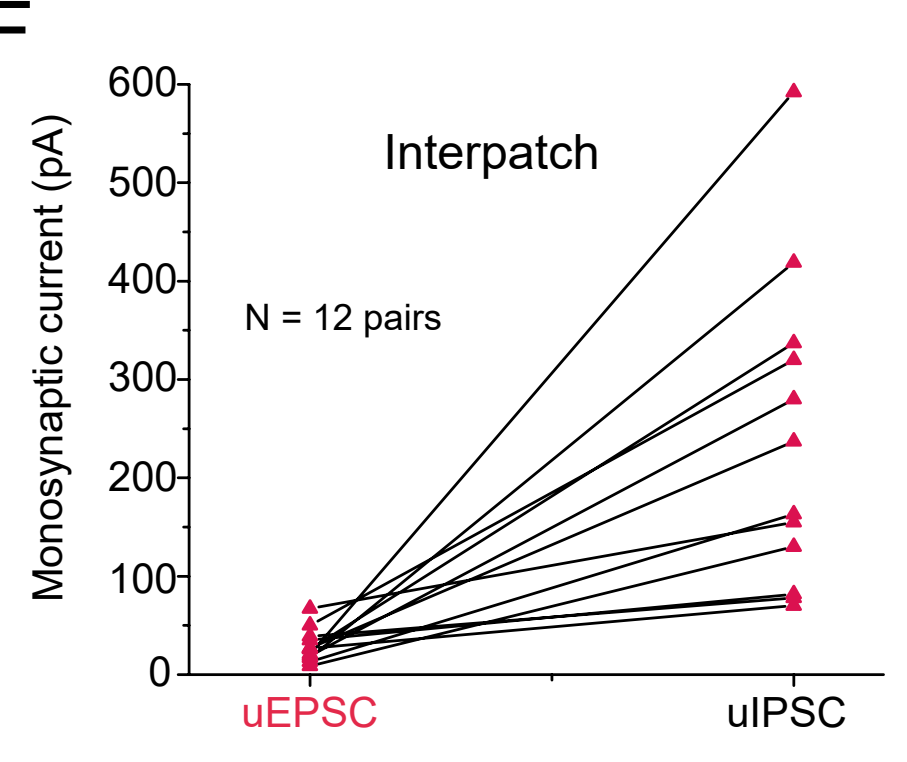

D

B Interpatch
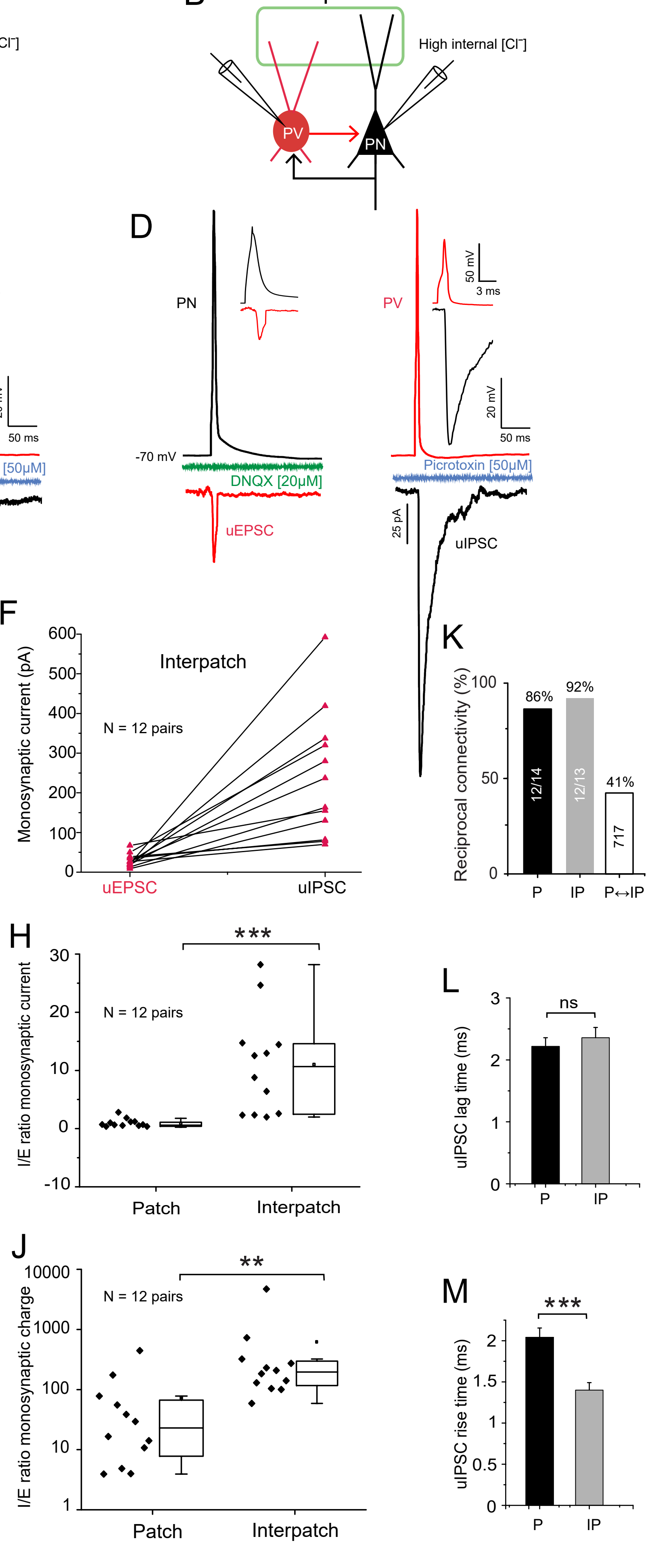

L

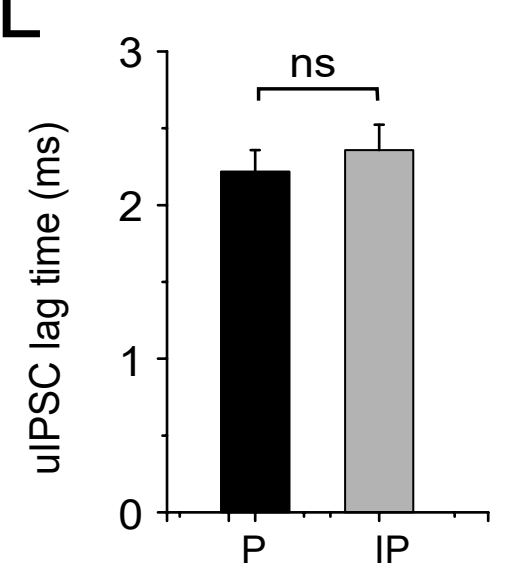

M

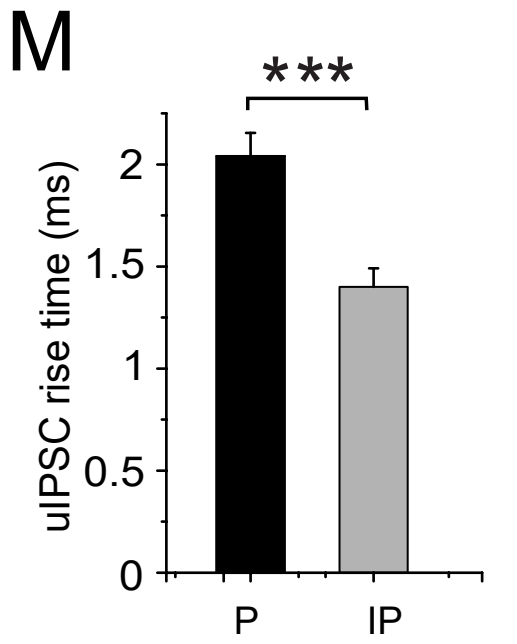

